\title{
Some Algebraic Aspects of the Inhomogeneous Six-Vertex Model
}

Vladimir V. BAZHANOV a, Gleb A. KOTOUSOV b, Sergii M. KOVAL a and Sergei L. LUKYANOV ${ }^{\text {cd }}$

a) Department of Theoretical Physics, Research School of Physics, Australian National University, Canberra, ACT 2601, Australia

E-mail: vladimir.bazhanov@anu.edu.au,sergii.koval@anu.edu.au

b) DESY, Theory Group, Notkestrasse 85, Hamburg, 22607, Germany

E-mail: gleb.kotousov@desy.de

c) NHETC, Department of Physics and Astronomy, Rutgers University, Piscataway, NJ 08855-0849, USA

E-mail: sergei@physics.rutgers.edu

d) Kharkevich Institute for Information Transmission Problems, Moscow, 127994, Russia

Received October 30, 2020, in final form February 26, 2021; Published online March 16, 2021

https://doi.org/10.3842/SIGMA.2021.025

\begin{abstract}
The inhomogeneous six-vertex model is a $2 D$ multiparametric integrable statistical system. In the scaling limit it is expected to cover different classes of critical behaviour which, for the most part, have remained unexplored. For general values of the parameters and twisted boundary conditions the model possesses $\mathrm{U}(1)$ invariance. In this paper we discuss the restrictions imposed on the parameters for which additional global symmetries arise that are consistent with the integrable structure. These include the lattice counterparts of $\mathcal{C}, \mathcal{P}$ and $\mathcal{T}$ as well as translational invariance. The special properties of the lattice system that possesses an additional $\mathcal{Z}_{r}$ invariance are considered. We also describe the Hermitian structures, which are consistent with the integrable one. The analysis lays the groundwork for studying the scaling limit of the inhomogeneous six-vertex model.
\end{abstract}

Key words: solvable lattice models; Bethe ansatz; Yang-Baxter equation; discrete symmetries; Hermitian structures

2020 Mathematics Subject Classification: 16T25; 52C26; 81T40; 82B20; 82B23

Dedicated to Professor Leon Armenovich Takhtajan on the occasion of his $70^{\text {th }}$ birthday

\section{Introduction}

The six-vertex model has played a central rôle in the theory of exactly solvable models in statistical mechanics. Its origins go back to the Pauling ice model [34] and it was later employed in the description of a two-dimensional ferroelectric (for a review see $[32,36]$ ). The free energy per site in the thermodynamic limit for a special "square ice" case was calculated by Lieb [31]. The result was then extended to the more general ferroelectric model by Sutherland, Yang and Yang [38, 39,41]. All these works dealt with the homogeneous statistical system defined on the square lattice. They used the technique pioneered by Bethe in [13], which allows one to compute the eigenvectors and eigenvalues of the transfer matrix. Later on, Baxter in the

This paper is a contribution to the Special Issue on Mathematics of Integrable Systems: Classical and Quantum in honor of Leon Takhtajan. The full collection is available at https://www.emis.de/journals/SIGMA/Takhtajan.html 


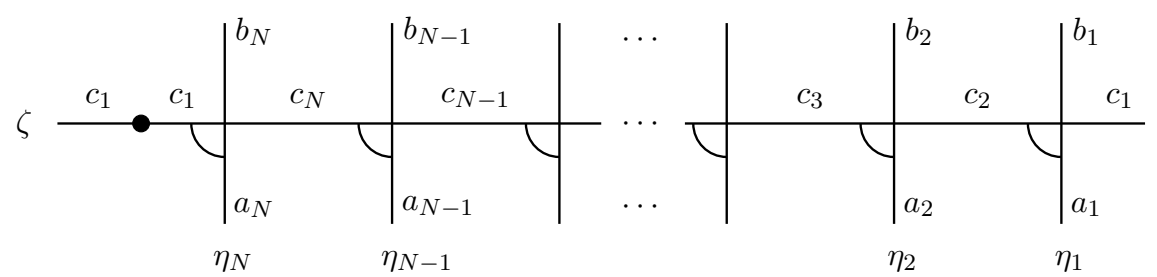

Figure 1. A graphical representation of the transfer matrix $(\mathbb{T}(\zeta))_{a_{N} a_{N-1} \ldots a_{1}}^{b_{N} b_{N-1} \ldots b_{1}}$ defined in (2.2). The black dot corresponds to the boundary twist $\omega^{c_{1}}$. Summation over the spin indices assigned to internal edges is assumed.

work [3] considered the case where the local Boltzmann weights vary from site to site. He found the most general inhomogeneous six-vertex model, which is still solvable by means of the Bethe ansatz technique. This was an important step that led to the concept of commuting transfer matrices. Subsequently, in his seminal paper on the exact solution of the eight-vertex model [4], Baxter developed the method of commuting transfer matrices, laying the foundation of the modern theory of integrable quantum systems. The approach was further extended by Faddeev, Sklyanin and Takhtajan within the quantum inverse scattering method (QISM) [37,40].

The local Boltzmann weights in the six-vertex model are expressed through the elements of the $R$-matrix, which is the trigonometric solution of the Yang-Baxter equation. The latter depends essentially on two parameters - the anisotropy and the spectral parameter. The inhomogeneous model considered in $[3,4]$ is defined on the square lattice, where each horizontal and vertical line is associated with its own variable. The local Boltzmann weights at each vertex have the same value of the anisotropy parameter, while the spectral parameter coincides with the ratio of the two variables assigned to the lines passing through the vertex. In this setting the row-to-row transfer matrix $\mathbb{T}$ for the lattice with $N$ vertical columns will depend on the variable $\zeta$, associated with the horizontal line, as well as the set $\left\{\eta_{1}, \eta_{2}, \ldots, \eta_{N}\right\}$, corresponding to the vertical lines (see Fig. 1). The Yang-Baxter equation implies that for the (quasi-)periodic boundary conditions the transfer matrices with different values of $\zeta$, but with the same sets $\left\{\eta_{J}\right\}$ form a commuting family

$$
\left[\mathbb{T}(\zeta), \mathbb{T}\left(\zeta^{\prime}\right)\right]=0 .
$$

The inhomogeneous six-vertex model turns out to be a multiparametric, integrable, statistical system that depends on the variables associated with the vertical lines, $\eta_{J}$, those labeling the horizontal lines and the anisotropy parameter. The latter will be referred to as $q$.

The most studied case of the model is when the set of local Boltzmann weights is the same at each vertex. Then $\eta_{1}=\eta_{2}=\cdots=\eta_{N}$ and the transfer matrix commutes with the spin $\frac{1}{2}$ Heisenberg $X X Z$ Hamiltonian. The anisotropy parameter of the spin chain, which is usually denoted as $\Delta$, is related to $q$ as $\Delta=\frac{1}{2}\left(q+q^{-1}\right)$. It determines the most interesting physical properties of the model. In particular when $-1<\Delta<1$ or, equivalently, $q$ is unimodular $(|q|=1)$ the system turns out to be critical and its scaling behaviour is governed by a conformal field theory (CFT) of a massless Gaussian field $[1,28,33]$. Perhaps the simplest inhomogeneous six-vertex model corresponds to a staggering of the variables associated with the vertical lines of the lattice such that $\eta_{1}=\eta_{3}=\cdots, \eta_{2}=\eta_{4}=\cdots$, while $\eta_{1} \neq \eta_{2}$ and a similar staggering for the variables assigned to the horizontal lines. In the case when $\eta_{1} / \eta_{2}>0$ and $|q|=1$, with a properly defined scaling limit where $\eta_{1} / \eta_{2} \rightarrow 0^{+}$(or $\left.+\infty\right)$ and $N \rightarrow \infty$, the lattice model exhibits universal behaviour that is described by the massive Thirring/sine-Gordon model $[17,27] .{ }^{1}$ The situation is different if the ratio of $\eta_{1}$ to $\eta_{2}$ is negative. For $\eta_{1} / \eta_{2}=-1$ and

\footnotetext{
${ }^{1}$ Generating a correlation length in a critical homogeneous lattice system via the introduction of an alternating set of inhomogeneities has been used in a variety of contexts see, e.g., [19,35].
} 
$|q|=1$ the system is critical and its important feature is the presence of a $\mathcal{Z}_{2}$ symmetry. For this reason the model is sometimes referred to as the $\mathcal{Z}_{2}$ invariant inhomogeneous six-vertex model. Various types of universal behaviour occur depending on whether $\arg \left(q^{2}\right) \in\left(0, \frac{\pi}{2}\right)$ or $\left(\frac{\pi}{2}, \pi\right)$. The latter case was considered in [24]. The former was the subject matter of the works $[8,16,20,21,23,25,26]$. It was originally observed in $[23,26]$ that there is a continuous component in the spectrum of conformal dimensions for the model. In [8] it was pointed out that the algebra of extended conformal symmetry underlying the critical behaviour is the $W_{\infty}$ algebra.

The identification of the critical surfaces in the space of couplings of the inhomogeneous six-vertex model as well as a description of the corresponding universality classes is, without doubt, an interesting problem. However, apart from the few cases mentioned above not much work has been done in this direction. An evident starting point to approach the problem would be to explore the restrictions on the parameters which give rise to extra symmetries in the model. The latter would include translational invariance as well as global involutions such as $\mathcal{C}, \mathcal{P}$ and $\mathcal{T}$, which are common attributes of a local QFT. There may also be other global symmetries, e.g., the $\mathcal{Z}_{2}$ invariance of the staggered model with $\eta_{J} / \eta_{1}=(-1)^{J-1}$. Of course, the identification of the universality classes requires more than just an analysis of the symmetries. The integrability of the model makes possible a detailed quantitative study of the scaling limit. The goal of this work is to describe the integrable structure underlying the general inhomogeneous six-vertex model as well as its interplay with the global symmetries.

The paper is organized as follows. In Section 2 we set-up the notation and give a short summary of the results of [3]. We also present the construction of the transfer matrix as well as its eigenstates (Bethe states) within the framework of the QISM. The commuting family for the inhomogeneous six-vertex model, apart from $\mathbb{T}(\zeta)$, contains other important members the so-called Baxter $Q$-operators [4]. Their construction along the lines of $[10,11]$ is presented in Section 3.

Section 4 is devoted to a discussion of the "charge" conjugation $\mathcal{C}$, parity inversion $\mathcal{P}$ and time reversal transformation $\mathcal{T}$. We describe the conditions under which these conjugations are consistent with the integrable structure in the sense that they preserve the family of commuting operators.

For a generic set of the inhomogeneities the transfer matrix and $Q$-operators are not Hermitian w.r.t. the standard matrix Hermitian conjugation. In Section 5 a family of Hermitian structures is introduced, which are also consistent with the integrable structure of the model. The norm of the Bethe states associated with these Hermitian structures can be computed via the formula originally conjectured by Gaudin, McCoy and $\mathrm{Wu}$ [22] for the homogeneous case and then extended and proven, using the formalism of the QISM, for the general inhomogeneous model by Korepin [30].

Further restrictions on the parameters of the model, which lead to the presence of additional global symmetries, are discussed in Sections 6 and 7. Among these is the invariance w.r.t. lattice translations. The latter appears when the number of columns of the lattice is divisible by some integer $r \geq 1$ and the inhomogeneities $\eta_{J}$ satisfy the $r$-site periodicity conditions $\eta_{J+r}=\eta_{J}$. In this case the commuting family of the model contains $r$ Hamiltonians, which are given by a sum of local operators. By the latter we mean that they are built from the local spin operators acting on $r+1$ consecutive sites of the lattice. Section 7 discusses the lattice system with the $\left\{\eta_{J}\right\}$ being further restricted such that $\eta_{J} / \eta_{1}=\mathrm{e}^{2 \pi \mathrm{i}(J-1) / r}$. Then the model possesses an extra $\mathcal{Z}_{r}$ cyclic group symmetry. The last section contains a collection of explicit formulae for the model with $r=1$ (homogeneous) and $r=2$ ( $\mathcal{Z}_{2}$ invariant). They lay the groundwork for a detailed analysis of the scaling limit for these two cases, which is performed in [9]. 


\section{The inhomogeneous six-vertex model}

Consider the square lattice with $N$ vertical columns. Each edge of the lattice can be in one of two states, labeled as \pm 1 . Introduce the $R$-matrix of the six-vertex model, whose non-zero entries are given by

$$
\begin{aligned}
& R_{++}^{++}(\zeta)=R_{--}^{--}(\zeta)=q+q^{-1} \zeta, \quad R_{+-}^{-+}(\zeta)=-\left(q-q^{-1}\right) \zeta, \\
& R_{+-}^{+-}(\zeta)=R_{-+}^{-+}(\zeta)=1+\zeta, \quad R_{-+}^{+-}(\zeta)=q-q^{-1},
\end{aligned}
$$

where $\zeta$ and $q$ are arbitrary parameters. Let $a_{1}, a_{2}, \ldots, a_{N}$ denote the states of a row of $N$ vertical edges of the lattice and $b_{1}, b_{2}, \ldots, b_{N}$ be the states on the row above. The elements of the row-to-row transfer matrix of the integrable inhomogeneous six-vertex model is defined as

$$
\begin{gathered}
(\mathbb{T}(\zeta))_{a_{N} a_{N-1} \ldots a_{1}}^{b_{N} b_{N-1} \ldots b_{1}}=q^{-\frac{N}{2}} \sum_{c_{1} c_{2} \cdots c_{N}= \pm} \omega^{c_{1}} R\left(q \zeta / \eta_{N}\right)_{c_{1} a_{N}}^{c_{N} b_{N}} R\left(q \zeta / \eta_{N-1}\right)_{c_{N} a_{N-1}}^{c_{N-1} b_{N-1}} \cdots \\
\cdots R\left(q \zeta / \eta_{2}\right)_{c_{3} a_{2}}^{c_{2} b_{2}} R\left(q \zeta / \eta_{1}\right)_{c_{2} a_{1}}^{c_{1} b_{1}}
\end{gathered}
$$

Here we have imposed twisted boundary conditions depending on the parameter $\omega$, while $\eta_{1}, \eta_{2}, \ldots, \eta_{N}$ are arbitrary parameters controlling the "inhomogeneity" of the model. The pre-

factor $q^{-\frac{N}{2}}$ has been introduced for convenience, to ensure the normalization condition (2.11) below. With the following graphical representation for the $R$-matrix

$$
R_{12}(q \zeta / \eta)_{a_{1} a_{2}}^{b_{1} b_{2}}=\zeta \frac{\underbrace{b_{2}}_{a_{2}} b_{1}}{\eta}
$$

the transfer matrix is depicted in Fig. 1.

The transfer matrix is an operator acting in the "quantum" space, which is formed by the direct product of $N$ two-dimensional spaces

$$
\mathscr{V}_{N}=\mathbb{C}_{N}^{2} \otimes \mathbb{C}_{N-1}^{2} \otimes \cdots \otimes \mathbb{C}_{1}^{2}
$$

Its diagonalization problem was solved by Baxter [3] via the coordinate Bethe ansatz. The eigenvectors with $M$ down spins have the form

$$
\Psi=\sum_{1 \leq x_{1}<x_{2}<\cdots<x_{M} \leq N} \Psi\left(x_{1}, \ldots, x_{M}\right) \sigma_{x_{M}}^{-} \cdots \sigma_{x_{1}}^{-}|0\rangle,
$$

where the (unnormalized) Bethe ansatz wave function reads as

$$
\Psi\left(x_{1}, \ldots, x_{M}\right)=\sum_{\hat{P}} A_{\hat{P}} \prod_{m=1}^{M} \phi_{\hat{P} m}\left(x_{m}\right)
$$

with suitably chosen coefficients $A_{\hat{P}}$ and functions $\phi_{m}(x)$. The vector $|0\rangle$ denotes the state with all spins up,

$$
|0\rangle=\underbrace{|\uparrow\rangle \otimes|\uparrow\rangle \otimes \cdots \otimes|\uparrow\rangle}_{N}
$$


and $\sigma_{J}^{z}, \sigma_{J}^{ \pm} \equiv \frac{1}{2}\left(\sigma_{J}^{x} \pm \mathrm{i} \sigma_{J}^{y}\right)$ stand for the Pauli matrices that act in the $J$-th factor of the tensor product (2.3). The summation is taken over all $M$ ! permutations $\hat{P}$ of the integers $(1,2, \ldots, M)$. Note that the number of down spins $M$ is simply related to the eigenvalue $S^{z}$ of the $z$-component of the total spin operator

$$
\mathbb{S}^{z}=\frac{1}{2} \sum_{J=1}^{N} \sigma_{J}^{z}: \quad \mathbb{S}^{z} \boldsymbol{\Psi}=S^{z} \mathbf{\Psi}, \quad M=\frac{1}{2} N-S^{z},
$$

which commutes with the transfer matrix.

To within an overall normalization of the wave function (2.5) the results of [3] can be summarized as follows:

$$
A_{\hat{P}}=\prod_{1 \leq j<m \leq M} \frac{q \zeta_{\hat{P} j}-q^{-1} \zeta_{\hat{P} m}}{\zeta_{\hat{P} j}-\zeta_{\hat{P} m}}
$$

where the set of complex numbers $\left\{\zeta_{j}\right\}_{j=1}^{M}$ satisfies the system of algebraic equations

$$
\prod_{J=1}^{N} \frac{\eta_{J}+q^{+1} \zeta_{m}}{\eta_{J}+q^{-1} \zeta_{m}}=-\omega^{2} q^{2 S^{z}} \prod_{j=1}^{M} \frac{\zeta_{j}-q^{+2} \zeta_{m}}{\zeta_{j}-q^{-2} \zeta_{m}}, \quad m=1,2, \ldots, M,
$$

and the functions $\phi_{m}(x)$ in $(2.5)$ have the form

$$
\phi_{m}(x)=-\mathrm{i} q^{\frac{1}{2}} \omega^{-1} \frac{\left(q-q^{-1}\right) \zeta_{m}}{q \eta_{x}+\zeta_{m}} \prod_{J=1}^{x-1} \frac{\eta_{J}+q \zeta_{m}}{q \eta_{J}+\zeta_{m}} .
$$

The corresponding eigenvalue of the transfer matrix,

$$
\mathbb{T}(\zeta) \Psi=T(\zeta) \Psi
$$

is given by $^{2}$

$$
\begin{aligned}
T(\zeta)= & \omega^{+1} q^{+S^{z}}\left(\prod_{J=1}^{N}\left(1+q^{-1} \zeta / \eta_{J}\right)\right) \prod_{m=1}^{M} \frac{\zeta_{m}-q^{+2} \zeta}{\zeta_{m}-\zeta} \\
& +\omega^{-1} q^{-S^{z}}\left(\prod_{J=1}^{N}\left(1+q^{+1} \zeta / \eta_{J}\right)\right) \prod_{m=1}^{M} \frac{\zeta_{m}-q^{-2} \zeta}{\zeta_{m}-\zeta} .
\end{aligned}
$$

It is easy to see from the definition (2.2) that the transfer matrix is an $N$-th order polynomial in the variable $\zeta$ satisfying the conditions

$$
\begin{aligned}
& \mathbb{T}(0)=\omega^{+1} q^{+\mathbb{S}^{z}}+\omega^{-1} q^{-\mathbb{S}^{z}}, \\
& \lim _{\zeta \rightarrow \infty} \zeta^{-N} \mathbb{T}(\zeta)=\left(\omega^{+1} q^{-\mathbb{S}^{z}}+\omega^{-1} q^{+\mathbb{S}^{z}}\right) \prod_{J=1}^{N} \eta_{J}^{-1} .
\end{aligned}
$$

Since a multiplication of all the $\eta_{J}$ by the same factor can be absorbed into a redefinition of the spectral parameter $\zeta$, we will always assume

$$
\prod_{J=1}^{N} \eta_{J}=1
$$

\footnotetext{
${ }^{2}$ Note that in the staggered case when $N$ is even and $\eta_{1}=\eta_{3}=\cdots=\eta_{N-1}, \eta_{2}=\eta_{4}=\cdots=\eta_{N}$ the diagonalization problem for $\mathbb{T}(\zeta)$ is significantly simplified. It becomes equivalent to that of the transfer matrix of the homogeneous six-vertex model on the $45^{\circ}$-rotated square lattice. The Bethe ansatz for this problem was worked out by Baxter in an unpublished work [2].
} 
The Bethe state (2.4) may also be constructed within the framework of the QISM [37,40]. Introduce the so-called monodromy matrix

$$
\boldsymbol{M}(\zeta) \equiv \boldsymbol{M}\left(\zeta \mid \eta_{N}, \eta_{N-1}, \ldots, \eta_{1}\right)=q^{-\frac{N}{2}} \boldsymbol{R}_{N}\left(q \zeta / \eta_{N}\right) \boldsymbol{R}_{N-1}\left(q \zeta / \eta_{N-1}\right) \cdots \boldsymbol{R}_{1}\left(q \zeta / \eta_{1}\right)
$$

where $\boldsymbol{R}_{m}$ is given by (2.1), but regarded as a two by two matrix

$$
\boldsymbol{R}_{m}(q \zeta)=\left(\begin{array}{cc}
q^{\frac{1}{2}\left(1+\sigma_{m}^{z}\right)}+q^{\frac{1}{2}\left(1-\sigma_{m}^{z}\right)} \zeta & -\left(q-q^{-1}\right) q \zeta \sigma_{m}^{-} \\
\left(q-q^{-1}\right) \sigma_{m}^{+} & q^{\frac{1}{2}\left(1-\sigma_{m}^{z}\right)}+q^{\frac{1}{2}\left(1+\sigma_{m}^{z}\right)} \zeta
\end{array}\right)
$$

whose elements act in the $m$-th factor of the tensor product (2.3). It is convenient to denote the entries of the monodromy matrix as

$$
\boldsymbol{M}(\zeta)=\left(\begin{array}{cc}
\hat{\mathrm{A}}(\zeta) & a(\zeta) \hat{\mathrm{B}}(\zeta) \\
d(\zeta) \hat{\mathrm{C}}(\zeta) & \hat{\mathrm{D}}(\zeta)
\end{array}\right)
$$

where $\hat{\mathrm{A}}, \hat{\mathrm{B}}, \hat{\mathrm{C}}, \hat{\mathrm{D}}$ are operators acting in $\mathscr{V}_{N}=\mathbb{C}_{N}^{2} \otimes \mathbb{C}_{N-1}^{2} \otimes \cdots \otimes \mathbb{C}_{1}^{2}$, while the functions

$$
a(\zeta)=-\mathrm{i} \omega^{+1} q^{+\frac{N+1}{2}} \prod_{J=1}^{N}\left(1+q^{-1} \zeta / \eta_{J}\right), \quad d(\zeta)=+\mathrm{i} \omega^{-1} q^{-\frac{N+1}{2}} \prod_{J=1}^{N}\left(1+q \zeta / \eta_{J}\right) .
$$

With the above notations, the transfer matrix (2.2) is given by

$$
\mathbb{T}(\zeta)=\operatorname{Tr}\left[\omega^{\sigma^{z}} \boldsymbol{M}(\zeta)\right]=\omega^{+1} \hat{\mathrm{A}}(\zeta)+\omega^{-1} \hat{\mathrm{D}}(\zeta),
$$

while the Bethe state (2.4)-(2.9), corresponding to the set $\left\{\zeta_{j}\right\}_{j=1}^{M}$ solving the Bethe ansatz equations, is expressed as

$$
\boldsymbol{\Psi}=\hat{\mathrm{B}}\left(\zeta_{M}\right) \cdots \hat{\mathrm{B}}\left(\zeta_{2}\right) \hat{\mathrm{B}}\left(\zeta_{1}\right)|0\rangle .
$$

Note that the following remarkable formula holds true:

$$
\hat{\mathrm{C}}\left(\zeta_{1}\right) \hat{\mathrm{C}}\left(\zeta_{2}\right) \cdots \hat{\mathrm{C}}\left(\zeta_{M}\right) \Psi=\mathfrak{K}[\boldsymbol{\Psi}]|0\rangle
$$

where we use the notation

$$
\begin{aligned}
\mathfrak{K}[\mathbf{\Psi}]=\left(q-q^{-1}\right)^{2 M} \prod_{m \neq j}^{M} \frac{q \zeta_{j}-q^{-1} \zeta_{m}}{\zeta_{m}-\zeta_{j}} \operatorname{det}[ & \delta_{j, m}\left(\kappa\left(\zeta_{j}\right)+\sum_{l=1}^{M} \frac{\left(q+q^{-1}\right) \zeta_{j} \zeta_{l}}{\left(q \zeta_{l}-q^{-1} \zeta_{j}\right)\left(q \zeta_{j}-q^{-1} \zeta_{l}\right)}\right) \\
& \left.-\frac{\left(q+q^{-1}\right) \zeta_{j} \zeta_{m}}{\left(q \zeta_{m}-q^{-1} \zeta_{j}\right)\left(q \zeta_{j}-q^{-1} \zeta_{m}\right)}\right]
\end{aligned}
$$

and

$$
\kappa(\zeta)=-\sum_{J=1}^{N} \frac{\zeta}{\eta_{J}\left(1+q^{-1} \zeta / \eta_{J}\right)\left(1+q^{+1} \zeta / \eta_{J}\right)} .
$$

This formula with all $\eta_{J}=1$ was originally conjectured by Gaudin, McCoy and Wu in [22]. Its generalization and proof for the inhomogeneous case was worked out by Korepin in [30]. 


\section{Q-operators}

The eigenvalues of the transfer matrix are polynomials in the variable $\zeta$. Indeed, its matrix elements defined through (2.2) and (2.1) are polynomials of $\zeta$ and its eigenvectors $(2.4)-(2.9)$ do not depend on this variable. The latter also implies that the matrices $\mathbb{T}(\zeta)$ with different values of $\zeta$ mutually commute. It turns out that the model possesses a larger commuting family which, together with the transfer matrix, contains other important members - the so-called Baxter $Q$-operators [4]. This section gives an overview of their construction as well as their main properties.

First note that, introducing the functions

$$
A_{+}(\zeta)=\prod_{m=1}^{M}\left(1-\zeta / \zeta_{m}\right), \quad f(\zeta)=\prod_{J=1}^{N}\left(1+\zeta / \eta_{J}\right),
$$

one can rewrite the expression for the eigenvalues $T(\zeta)(2.10)$ in the form

$$
T(\zeta) A_{+}(\zeta)=\omega^{+1} q^{+S^{z}} f\left(q^{-1} \zeta\right) A_{+}\left(q^{+2} \zeta\right)+\omega^{-1} q^{-S^{z}} f\left(q^{+1} \zeta\right) A_{+}\left(q^{-2} \zeta\right) .
$$

Since $T(\zeta)$ is a polynomial, the r.h.s. of the last relation must vanish at the zeroes of $A_{+}(\zeta)$, i.e., when $\zeta=\zeta_{m}$, so that

$$
\omega^{+1} q^{+S^{z}} f\left(q^{-1} \zeta_{m}\right) A_{+}\left(q^{+2} \zeta_{m}\right)+\omega^{-1} q^{-S^{z}} f\left(q^{+1} \zeta_{m}\right) A_{+}\left(q^{-2} \zeta_{m}\right)=0, \quad m=1,2, \ldots, M .
$$

These are precisely the Bethe ansatz equations (2.8). The functional relation (3.2) implies the existence of an operator $\mathbb{A}_{+}(\zeta)$, with eigenvalues $A_{+}(\zeta)(3.1)$, that commutes with the transfer matrix $\mathbb{T}(\zeta)$ and satisfies the operator version of (3.2). ${ }^{3}$ Actually there are two such operators, which we denote as $\mathbb{A}_{ \pm}(\zeta)$, that satisfy the operator relations of this type

$$
\mathbb{T}(\zeta) \mathbb{A}_{ \pm}(\zeta)=q^{ \pm 2 \mathbb{P}} f\left(q^{-1} \zeta\right) \mathbb{A}_{ \pm}\left(q^{+2} \zeta\right)+q^{\mp 2 \mathbb{P}} f\left(q^{+1} \zeta\right) \mathbb{A}_{ \pm}\left(q^{-2} \zeta\right)
$$

where for convenience we have used the notation

$$
q^{2 \mathbb{P}}=\omega q^{\mathbb{S}^{z}}
$$

Note that equation (3.3) can be rewritten in a way so that it has the same form for the "+" and "-" cases. Introducing the operators $\mathbb{Q}_{ \pm}(\zeta)$, which differ from $\mathbb{A}_{ \pm}(\zeta)$ by simple factors involving fractional powers of $\zeta$,

$$
\mathbb{Q}_{ \pm}(\zeta)=\zeta^{ \pm \mathbb{P}} \mathbb{A}_{ \pm}(\zeta)
$$

one has

$$
\mathbb{T}(\zeta) \mathbb{Q}_{ \pm}(\zeta)=f\left(q^{-1} \zeta\right) \mathbb{Q}_{ \pm}\left(q^{+2} \zeta\right)+f\left(q^{+1} \zeta\right) \mathbb{Q}_{ \pm}\left(q^{-2} \zeta\right)
$$

The latter is the famous Baxter "TQ-relation" [4]. We prefer to work with the operators $\mathbb{A}_{ \pm}(\zeta)$ as their matrix elements and eigenvalues are polynomials in $\zeta$.

Similar to $\mathbb{T}(\zeta)$, which can be expressed as a trace of a $2 \times 2$ matrix (2.17), the operators $\mathbb{A}_{ \pm}(\zeta)$ are also constructed as traces of certain monodromy matrices. However, this time the trace is taken over infinite dimensional representations of the so-called $q$-oscillator algebra. The latter arises as an evaluation representation of the Borel subalgebra of the quantum affine

\footnotetext{
${ }^{3}$ In this paper we use blackboard bold symbols such as $\mathbb{T}, \mathbb{A}_{ \pm}, \mathbb{Q}_{ \pm}, \mathbb{S}^{z}, \ldots$ to denote the mutually commuting operators acting in the space $\mathscr{V}_{N}(2.3)$. For the operators acting in the same space which do not belong to the commuting family, say the entries $\hat{A}, \hat{B}, \hat{C}, \hat{D}$ of the monodromy matrix (2.15), the "hat" notation is employed.
} 
algebra $U_{q}\left(\widehat{\mathfrak{s l}}_{2}\right)$. The required $R$-matrices (see (3.6) below) are obtained by a suitable specialization of the universal $R$-matrix [29]. Originally these calculations were performed in the context of the quantum $\mathrm{KdV}$ theory $[10,11]$, but the same procedure can be readily applied to the inhomogeneous six-vertex model.

The $q$-oscillator algebra is generated by the elements $\mathcal{H}$ and $\mathcal{E}_{ \pm}$satisfying the commutation relations

$$
\left[\mathcal{H}, \mathcal{E}_{ \pm}\right]= \pm 2 \mathcal{E}_{ \pm}, \quad q \mathcal{E}_{+} \mathcal{E}_{-}-q^{-1} \mathcal{E}_{-} \mathcal{E}_{+}=\frac{1}{q-q^{-1}}
$$

The basic building blocks for the construction of $\mathbb{A}_{ \pm}$are the $2 \times 2$ matrices

$$
\begin{aligned}
\mathcal{R}_{m}^{( \pm)}(\zeta)= & q^{ \pm \frac{\mathcal{H} \otimes \sigma_{m}^{z}}{2}}+\left(q-q^{-1}\right)\left(\mathcal{E}_{\mp} \otimes \sigma_{m}^{+}-\zeta \mathcal{E}_{ \pm} \otimes \sigma_{m}^{-}\right) \\
& +\zeta\left(q-q^{-1}\right) q^{-\frac{\mathcal{H}}{2}}\left(\mathcal{E}_{+} \mathcal{E}_{-}-\mathcal{E}_{-} \mathcal{E}_{+}\right) \otimes \sigma_{m}^{\mp} \sigma_{m}^{ \pm}
\end{aligned}
$$

which act in the $m$-th component of the tensor product (2.3) and whose entries involve the formal generators (3.5). Let $\rho_{ \pm}$be representations of the $q$-oscillator algebra such that the traces

$$
\operatorname{Tr}_{\rho_{ \pm}}\left[q^{ \pm 2 P \mathcal{H}}\right] \neq 0 \quad \text { with } \quad\left|q^{2 P}\right|>1
$$

exist and are non-vanishing. Then, using the notation (3.4) and assuming that the twist parameter $\omega$ is such that

$$
|\omega|>\max \left(\left|q^{N / 2}\right|,\left|q^{-N / 2}\right|\right)
$$

one can introduce the operators

$$
\mathbb{Z}_{ \pm}=\operatorname{Tr}_{\rho_{ \pm}}\left[q^{ \pm 2 \mathbb{P} \mathcal{H}}\right]
$$

that act in the quantum space $\mathscr{V}_{N}(2.3)$. With these preparations, define

$$
\mathbb{A}_{ \pm}(\zeta)=\mathbb{Z}_{ \pm}^{-1} \operatorname{Tr}_{\rho_{ \pm}}\left[\omega^{ \pm \mathcal{H}} \mathcal{R}_{N}^{( \pm)}\left(\zeta / \eta_{N}\right) \mathcal{R}_{N-1}^{( \pm)}\left(\zeta / \eta_{N-1}\right) \cdots \mathcal{R}_{1}^{( \pm)}\left(\zeta / \eta_{1}\right)\right]
$$

It is easy to see that such operators commute with $\mathbb{S}^{z}$ and, therefore, with $\mathbb{Z}_{ \pm}$. As was first pointed out in [10], the (normalized) trace in (3.9) is completely determined by the commutation relations (3.5) and the cyclic property of the trace, so that the specific choice of the representations $\rho_{ \pm}$is not significant as long as the traces (3.8) exist and are non-vanishing. This way it is possible to define $\mathbb{A}_{ \pm}(\zeta) \in \operatorname{End}\left(\mathscr{V}_{N}\right)$ for all complex $\omega$ (except some discrete set of isolated points, see below) through analytic continuation, despite that the definition (3.9) applies literally to the case (3.7) only.

Following the line of $[11]$, one can show that the operators $\mathbb{A}_{ \pm}(\zeta)$ commute with the transfer matrix and among themselves for different values of the spectral parameter:

$$
\left[\mathbb{A}_{ \pm}\left(\zeta_{1}\right), \mathbb{T}\left(\zeta_{2}\right)\right]=\left[\mathbb{A}_{ \pm}\left(\zeta_{1}\right), \mathbb{A}_{ \pm}\left(\zeta_{2}\right)\right]=\left[\mathbb{A}_{ \pm}\left(\zeta_{1}\right), \mathbb{A}_{\mp}\left(\zeta_{2}\right)\right]=0 .
$$

Furthermore they satisfy a number of important operator valued relations, which are derived algebraically from the decomposition properties of products of representations of the $q$-oscillator algebra. This includes the quantum Wronskian relation

$$
\left(q^{+2 \mathbb{P}}-q^{-2 \mathbb{P}}\right) f(\zeta)=q^{+2 \mathbb{P}} \mathbb{A}_{+}\left(q^{+1} \zeta\right) \mathbb{A}_{-}\left(q^{-1} \zeta\right)-q^{-2 \mathbb{P}} \mathbb{A}_{-}\left(q^{+1} \zeta\right) \mathbb{A}_{+}\left(q^{-1} \zeta\right)
$$

as well as

$$
\left(q^{+2 \mathbb{P}}-q^{-2 \mathbb{P}}\right) \mathbb{T}(\zeta)=q^{+4 \mathbb{P}} \mathbb{A}_{+}\left(q^{+2} \zeta\right) \mathbb{A}_{-}\left(q^{-2} \zeta\right)-q^{-4 \mathbb{P}} \mathbb{A}_{-}\left(q^{+2} \zeta\right) \mathbb{A}_{+}\left(q^{-2} \zeta\right) .
$$

As a simple corollary of the above two formulae, the operators $\mathbb{A}_{ \pm}$satisfy (3.3). 
From the definition (3.9) it follows that the eigenvalues of $\mathbb{A}_{ \pm}(\zeta)$ are polynomials

$$
A_{ \pm}(\zeta)=\prod_{m=1}^{M_{ \pm}}\left(1-\zeta / \zeta_{m}^{( \pm)}\right)
$$

of degree

$$
M_{ \pm}=\frac{1}{2} N \mp S^{z}
$$

Their roots $\zeta_{m}^{( \pm)}$are determined by the Bethe ansatz equations

$$
\left.\frac{f\left(\zeta_{m}^{( \pm)} q^{+1}\right)}{f\left(\zeta_{m}^{( \pm)} q^{-1}\right)}=-\omega^{ \pm 2} q^{ \pm 2 S^{z}} \frac{A_{ \pm}\left(\zeta_{m}^{( \pm)} q^{+2}\right)}{A_{ \pm}\left(\zeta_{m}^{( \pm)} q^{-2}\right)}, \quad m=1,2, \ldots, M_{\partial}\right) .
$$

Formula (3.11) implies that

$$
\mathbb{A}_{ \pm}(\zeta) \rightarrow \begin{cases}1 & \text { as } \quad \zeta \rightarrow 0 \\ \zeta^{M_{ \pm}} \mathbb{A}_{ \pm}^{(\infty)} & \text { as } \quad \zeta \rightarrow+\infty\end{cases}
$$

where the operators $\mathbb{A}_{ \pm}^{(\infty)}$ act in $\mathscr{V}_{N}(2.3)$ and their eigenvalues on the Bethe state (2.18) read as

$$
A_{ \pm}^{(\infty)}=\prod_{j=1}^{M_{ \pm}}\left(-\zeta^{( \pm)}\right)^{-1}
$$

Combining (3.12) with (3.10) one obtains

$$
\mathbb{A}_{+}^{(\infty)} \mathbb{A}_{-}^{(\infty)}=\frac{1-\omega^{2} q^{2 \mathbb{S}^{z}}}{q^{2 \mathbb{S}^{z}}-\omega^{2}}
$$

It should be pointed out that, defined through equation (3.9), the operators $\mathbb{A}_{ \pm}(\zeta)$ obey the normalization condition $\mathbb{A}_{ \pm}(0)=\hat{\mathbf{1}}$. However, in the subspace with fixed $S^{z}$ and when $\omega= \pm q^{-S^{z}+m}$ with $m= \pm 1, \pm 2, \ldots$ the Bethe roots $\left\{\zeta_{m}\right\}$ for some of the eigenvalues of $\mathbb{A}_{ \pm}$ may become zero. In this case, as it follows from (3.11), the normalization imposed by (3.9) is not suitable and that formula requires modification. As our considerations are not sensitive to this subtlety, we will continue to use (3.9) and assume that (3.11) always holds.

Finally note that for $S^{z}=0$ both $\mathbb{A}_{ \pm}(\zeta)$ tend to the same operator $\mathbb{A}(\zeta)$ as $\omega \rightarrow 1$. A careful taking of this limit in equation (3.9) leads to a generalization of Baxter's formula for the matrix elements of the $Q$-operator of the zero-field six-vertex model in the sector with $S^{z}=0$ :

$$
(\mathbb{A}(\zeta))_{a_{N}, a_{N-1}, \ldots, a_{1}}^{b_{N}, b_{N-1}, \ldots, b_{1}}=q^{\frac{1}{4} \sum_{1 \leq J<K \leq N}\left(a_{K} b_{J}-a_{J} b_{K}\right)} \prod_{J=1}^{N}\left(-\zeta / \eta_{J}\right)^{\frac{1}{4}\left(1-a_{J}\right)\left(1+b_{J}\right)} .
$$

In the homogeneous case, when all the $\eta_{J}=1$, the last formula reduces to equation (101) of [5]. Note that the operator (3.13) is still normalized as $\mathbb{A}(0)=1$, which may not be immediately obvious.

\section{$4 \mathcal{C}, \mathcal{P}$ and $\mathcal{T}$ conjugations}

So far we have briefly reviewed the formal algebraic aspects of the diagonalization problem for the commuting family of operators in the general inhomogeneous six-vertex model. All the parameters $\left\{\eta_{J}\right\}, q$ and $\omega$ were assumed to be generic complex numbers. Though the constraint (2.12) was imposed, it does not reduce the generality, as it can always be achieved via 
a redefinition of the spectral parameter $\zeta$. In this section we introduce three global conjugations, which are similar to the charge conjugation $\mathcal{C}$, parity inversion $\mathcal{P}$, and time reversal transformation $\mathcal{T}$ from quantum field theory. We describe the restrictions on the parameters such that the global involutions are consistent with the integrable structure, in the sense that they preserve the family of commuting operators.

Let us first consider parity inversion. This transformation may be defined via the following adjoint action on the local spin operators

$$
\hat{\mathcal{P}} \sigma_{J}^{A} \hat{\mathcal{P}}=\left(\eta_{N+1-J}\right)^{+\frac{1}{2} \sigma_{N+1-J}^{z}} \sigma_{N+1-J}^{A}\left(\eta_{N+1-J}\right)^{-\frac{1}{2} \sigma_{N+1-J}^{z}}, \quad A=x, y, z, \quad J=1, \ldots, N,
$$

supplemented by the condition

$$
\hat{\mathcal{P}}|0\rangle=|0\rangle,
$$

where $|0\rangle$ stands for the pseudovacuum $(2.6) .{ }^{4}$ Its matrix elements read as

$$
(\hat{\mathcal{P}})_{a_{N} a_{N-1} \cdots a_{1}}^{b_{N} b_{N-1} \cdots b_{1}}=\delta_{a_{N}}^{b_{1}} \delta_{a_{N-1}}^{b_{2}} \cdots \delta_{a_{1}}^{b_{N}} \prod_{J=1}^{N} \eta_{J}^{a_{J} / 2} .
$$

In order for $\hat{\mathcal{P}}^{2}=\hat{\mathbf{1}}$, one must require that

$$
\eta_{J}=\left(\eta_{N+1-J}\right)^{-1}, \quad J=1,2, \ldots, N .
$$

Then the action of the parity conjugation on the transfer matrix and $\mathbb{A}_{ \pm}(\zeta)$ is given by

$$
\begin{aligned}
& \hat{\mathcal{P}} \mathbb{T}(\zeta \mid \omega) \hat{\mathcal{P}}=\zeta^{N} \mathbb{T}\left(\zeta^{-1} \mid \omega^{-1}\right), \\
& \hat{\mathcal{P}} \mathbb{A}_{ \pm}(\zeta \mid \omega) \hat{\mathcal{P}}=\zeta^{\left(\frac{N}{2} \mp \mathbb{S}^{z}\right)} \mathbb{A}_{ \pm}\left(\zeta^{-1} \mid \omega^{-1}\right)\left[\mathbb{A}_{ \pm}^{(\infty)}\left(\omega^{-1}\right)\right]^{-1}
\end{aligned}
$$

where we explicitly indicate the dependence on the twist parameter $\omega$. These relations may be deduced directly from the definition of $\mathbb{T}(\zeta)$ and $\mathbb{A}_{ \pm}(\zeta)$. For instance, the first equality follows from (2.17) and (2.13) as well as the simple property

$$
\left(\sigma^{x} \otimes \zeta^{\frac{1}{2} \sigma_{m}^{z}}\right)\left(\boldsymbol{R}_{m}(q \zeta)\right)^{t}\left(\sigma^{x} \otimes \zeta^{\frac{1}{2} \sigma_{m}^{z}}\right)^{-1}=\zeta \boldsymbol{R}_{m}\left(q \zeta^{-1}\right)
$$

where $\left(\boldsymbol{R}_{m}(q \zeta)\right)^{t}$ stands for the transpose of the $2 \times 2$ operator valued matrix (2.14). Since $\mathbb{T}(\zeta \mid \omega)$ and $\mathbb{A}_{ \pm}(\zeta \mid \omega)$ do not commute amongst themselves for different values of the twist parameter $\omega$, formula (4.4) shows that unless $\omega^{2}=1$, the parity inversion does not preserve the commuting family of operators.

The situation is similar for the charge conjugation. Its generator may be introduced as

$$
\hat{\mathcal{C}}=\prod_{J=1}^{N}\left(\eta_{J}\right)^{\frac{1}{2} \sigma_{J}^{z}} \sigma_{J}^{x},
$$

whose adjoint action on the local spin operators is given by

$$
\hat{\mathcal{C}} \sigma_{J}^{ \pm} \hat{\mathcal{C}}=\eta_{J}^{\mp 1} \sigma_{J}^{\mp}, \quad \hat{\mathcal{C}} \sigma_{J}^{z} \hat{\mathcal{C}}=-\sigma_{J}^{z}, \quad J=1, \ldots, N .
$$

For arbitrary complex values of the inhomogeneities one has

$$
\hat{\mathcal{C}} \mathbb{T}(\zeta \mid \omega) \hat{\mathcal{C}}=\mathbb{T}\left(\zeta \mid \omega^{-1}\right), \quad \hat{\mathcal{C}} \mathbb{A}_{ \pm}(\zeta \mid \omega) \hat{\mathcal{C}}=\mathbb{A}_{\mp}\left(\zeta \mid \omega^{-1}\right) .
$$

\footnotetext{
${ }^{4}$ The definition of the generator $\hat{\mathcal{P}}$ contains an ambiguity in the choice of the overall $\operatorname{sign} \hat{\mathcal{P}} \mapsto c_{N} \hat{\mathcal{P}}$ with $c_{N}^{2}=1$. In this work it is fixed by the condition (4.1). In [9] the sign factor is chosen differently. The same also applies to the generator of the charge conjugation $\hat{\mathcal{C}}(4.5)$.
} 
The proof of the first equality is based on the relation

$$
\left(\sigma^{x} \otimes \zeta^{-\frac{1}{2} \sigma_{m}^{z}} \sigma_{m}^{y}\right) \boldsymbol{R}_{m}(\zeta)\left(\sigma^{x} \otimes \zeta^{-\frac{1}{2} \sigma_{m}^{z}} \sigma_{m}^{y}\right)^{-1}=\boldsymbol{R}_{m}(\zeta)
$$

Though the $\mathcal{C}$ and $\mathcal{P}$ transformations do not respect the integrable structure themselves for $\omega^{2} \neq 1$, by combining them together one gets

$$
\begin{aligned}
& \hat{\mathcal{C}} \hat{\mathcal{P}} \mathbb{T}(\zeta) \hat{\mathcal{C}} \hat{\mathcal{P}}=\zeta^{N} \mathbb{T}\left(\zeta^{-1}\right), \\
& \hat{\mathcal{C}} \hat{\mathcal{P}} \mathbb{A}_{ \pm}(\zeta) \hat{\mathcal{C}} \hat{\mathcal{P}}=\zeta^{\frac{N}{2} \mp \mathbb{S}^{z}} \mathbb{A}_{\mp}\left(\zeta^{-1}\right)\left[\mathbb{A}_{ \pm}^{(\infty)}\right]^{-1}
\end{aligned}
$$

provided that the conditions (4.3) are imposed. Here all the original and transformed operators correspond to the same value of $\omega$ so that they commute with each other and have the same set of eigenvectors. This has an important consequence. Since the $\mathcal{C P}$ conjugation intertwines the sectors with $S^{z}$ and $-S^{z}$ it is always possible to focus on the case with $S^{z} \geq 0$. Further, the second line of equation (4.7) implies that for the construction of the Bethe states (2.18) in the sector with arbitrary $S^{z}$ it is sufficient to consider the operator $\mathbb{A}_{+}(\zeta)$. For these reasons we focus on the zeroes of the eigenvalues of $\mathbb{A}_{+}$and use the notation $\zeta_{m} \equiv \zeta_{m}^{(+)}$and $M \equiv M_{+} \leq \frac{N}{2}$.

Up to this point there was no essential need to impose any reality conditions on the parameters of the model. However, for the time reversal transformation, the reality conditions become crucial. Having in mind applications to the $\mathcal{Z}_{2}$ invariant model studied in $[8,9,16,20,21,23,25,26]$, we will assume that $q$ and $\omega$ are unimodular and parameterize them as

$$
q=\mathrm{e}^{\mathrm{i} \gamma}, \quad 0<\gamma<\pi,
$$

and

$$
\omega=\mathrm{e}^{\mathrm{i} \pi \mathrm{k}}, \quad-\frac{1}{2}<\mathrm{k} \leq \frac{1}{2} .
$$

The $R$-matrix then obeys the property

$$
\left(\sigma^{x} \otimes \sigma^{x}\right)(\boldsymbol{R}(q \zeta))^{*}\left(\sigma^{x} \otimes \sigma^{x}\right)=q^{-1} \zeta^{*} \boldsymbol{R}\left(q / \zeta^{*}\right) .
$$

As for the inhomogeneities, three cases will be considered:

(i) All $\eta_{J}$ are unimodular complex numbers satisfying (4.3):

$$
\eta_{J}^{*}=\eta_{J}^{-1}=\eta_{N+1-J} .
$$

(ii) All the inhomogeneities are real:

$$
\eta_{J}^{*}=\eta_{J}=\left(\eta_{N+1-J}\right)^{-1}
$$

(iii) In the third case we take the number of columns in the lattice to be even and divide the inhomogeneities into two groups: $\left\{\eta_{J}\right\}_{J=1}^{N / 2}$ and $\left\{\eta_{N / 2+J}\right\}_{J=1}^{N / 2}$. The first group $\left\{\eta_{J}\right\}_{J=1}^{N / 2}$ are taken to have the same absolute value $\Lambda>0$, while for the other group $\left|\eta_{N / 2+1}\right|=\cdots=$ $\left|\eta_{N}\right|=\Lambda^{-1}$. The arguments of $\eta_{J}$ and $\eta_{N / 2+J}$ are chosen to be the same

$$
\eta_{J}=\Lambda^{-1} \omega_{J}, \quad \eta_{N / 2+J}=\Lambda \omega_{J}, \quad J=1,2, \ldots, N / 2
$$

where $\left\{\omega_{J}\right\}_{J=1}^{N / 2}$ are unimodular complex numbers. Notice that the constraint (4.3), which is always assumed, implies

$$
\omega_{J}=\left(\omega_{J}^{*}\right)^{-1}=\left(\omega_{N / 2+1-J}\right)^{-1} .
$$


The time reversal transformation is realized as an anti-unitary operator acting on an arbitrary state $\boldsymbol{\psi} \in \mathscr{V}_{N}$ as

$$
\hat{\mathcal{T}} \boldsymbol{\psi}=\hat{\mathrm{U}} \boldsymbol{\psi}^{*},
$$

where the asterisk $(*)$ stands for complex conjugation. The matrix $\hat{U}$ satisfies the condition

$$
\hat{\mathrm{U}}^{*}=\hat{\mathrm{U}}^{-1}
$$

which ensures that $\hat{\mathcal{T}}^{2}=\hat{\mathbf{1}}$. For the first case (4.8) we set the matrix $\hat{U}$ to be

$$
\eta_{J}^{*}=\eta_{J}^{-1}=\eta_{N+1-J}: \quad \hat{\mathrm{U}}=\prod_{J=1}^{N} \sigma_{J}^{x} .
$$

Then it follows that

$$
\begin{aligned}
& \hat{\mathcal{T}} \mathbb{T}(\zeta) \hat{\mathcal{T}}=\left(\zeta^{*}\right)^{N} \mathbb{T}\left(\left(\zeta^{*}\right)^{-1}\right), \\
& \hat{\mathcal{T}} \mathbb{A}_{ \pm}(\zeta) \hat{\mathcal{T}}=\left(\zeta^{*}\right)^{\frac{N}{2} \mp \mathbb{S}^{z}} \mathbb{A}_{\mp}\left(\left(\zeta^{*}\right)^{-1}\right)\left[\mathbb{A}_{\mp}^{(\infty)}\right]^{-1},
\end{aligned}
$$

i.e., defined in this way the time reversal transformation preserves the integrable structure of the model similar to $\mathcal{C P}$. Combining all three transformations (4.6), (4.4) and (4.15) together, yields

$$
\hat{\mathcal{C}} \hat{\mathcal{P}} \hat{\mathcal{T}} \mathbb{A}_{ \pm}(\zeta) \hat{\mathcal{C}} \hat{\mathcal{P}} \hat{\mathcal{T}}=\mathbb{A}_{ \pm}\left(\zeta^{*}\right), \quad \hat{\mathcal{C}} \hat{\mathcal{P}} \hat{\mathcal{T}} \mathbb{T}(\zeta) \hat{\mathcal{C}} \hat{\mathcal{P}} \hat{\mathcal{T}}=\mathbb{T}\left(\zeta^{*}\right) .
$$

For the Bethe states, the above equation together with (3.1) implies that the sets $\left\{\zeta_{m}\right\}$ corresponding to $\boldsymbol{\Psi}$ and $\hat{\mathcal{C}} \hat{\mathcal{P}} \hat{\mathcal{T}} \boldsymbol{\Psi}$ are complex conjugate to each other. Notice that the phase assignment in (2.9) and consequently in (2.16) has been chosen in such a way that

$$
\hat{\mathcal{C}} \hat{\mathcal{P}} \hat{\mathcal{T}} \Psi\left(\left\{\zeta_{j}\right\}\right)=\Psi\left(\left\{\zeta_{j}^{*}\right\}\right) .
$$

In the second case $(\mathrm{ii})$ it is also possible to introduce the time-reversal symmetry in such a way that relations (4.15) are preserved. To proceed we will need to establish how the transfer matrix and, more generally, the monodromy matrix $\boldsymbol{M}\left(\zeta \mid \eta_{N}, \eta_{N-1}, \ldots, \eta_{1}\right)(2.13)$, behaves under an interchange of the inhomogeneities. The eigenvalues of the transfer matrix (2.10) depend on $\left\{\eta_{J}\right\}$ both explicitly and implicitly via the Bethe roots which satisfy equation (2.8). In both cases the inhomogeneities enter only through symmetric combinations, so that the eigenvalues are symmetric functions of them. Therefore two transfer matrices obtained from each other by a permutation of the same set of inhomogeneities must be connected by a similarity transformation in the quantum space $\mathscr{V}_{N}$. In fact, the same holds true for all the entries of the monodromy matrix as well. The group of permutations of the ordered set $\left(\eta_{J}\right)$ is generated by elementary permutations of neighbouring pairs:

$$
\left(\eta_{N}, \ldots, \eta_{n+1}, \eta_{n}, \ldots, \eta_{1}\right) \mapsto\left(\eta_{N}, \ldots, \eta_{n}, \eta_{n+1}, \ldots, \eta_{1}\right) .
$$

The latter are realized through the relation

$$
\begin{aligned}
& \boldsymbol{M}\left(\zeta \mid \eta_{N}, \ldots, \eta_{n+1}, \eta_{n}, \ldots, \eta_{1}\right) \check{\boldsymbol{R}}_{n+1, n}\left(\eta_{n+1} / \eta_{n}\right) \\
& \quad=\check{\boldsymbol{R}}_{n+1, n}\left(\eta_{n+1} / \eta_{n}\right) \boldsymbol{M}\left(\zeta \mid \eta_{N}, \ldots, \eta_{n}, \eta_{n+1}, \ldots, \eta_{1}\right),
\end{aligned}
$$

which is a simple consequence of the Yang-Baxter equation. Here $\check{\boldsymbol{R}}_{n+1, n}$ is expressed in terms of the $R$-matrix (2.1) as

$$
\check{\boldsymbol{R}}_{n+1, n}(\zeta)=\frac{1}{q-q^{-1} \zeta} \boldsymbol{R}_{n+1, n}(-\zeta) \boldsymbol{P}_{n+1, n}
$$


with $\boldsymbol{P}_{n+1, n}$ standing for the operator that permutes the $(n+1)$-th and $n$-th factor in the tensor product (2.3). For the operation that completely reverses the order of the $\left\{\eta_{J}\right\}$ it is easy to see that

$$
\boldsymbol{M}\left(\zeta \mid \eta_{N}, \eta_{N-1}, \ldots, \eta_{1}\right) \mapsto \boldsymbol{M}\left(\zeta \mid \eta_{1}, \eta_{2}, \ldots, \eta_{N}\right)=\hat{\mathrm{S}}_{2}^{-1} \boldsymbol{M}\left(\zeta \mid \eta_{N}, \eta_{N-1}, \ldots, \eta_{1}\right) \hat{\mathrm{S}}_{2}
$$

where the matrix $\hat{\mathrm{S}}_{2} \in \operatorname{End}\left(\mathscr{V}_{N}\right)$ is given by the ordered product

$$
\hat{\mathrm{S}}_{2}=\prod_{m=2}^{N}\left[\prod_{n=N-m+1}^{\curvearrowleft} \check{\boldsymbol{R}}_{n+1, n}\left(\eta_{m} / \eta_{n+m-N}\right)\right] \text {. }
$$

The time-reversal transformation corresponding to (4.9) is defined through (4.12) with

$$
\eta_{J}^{*}=\eta_{J}=\left(\eta_{N+1-J}\right)^{-1}: \quad \hat{\mathrm{U}}=\hat{\mathrm{S}}_{2} \prod_{J=1}^{N} \sigma_{J}^{x}
$$

One can check that the condition (4.13) is satisfied, and that equations (4.15), (4.16) remain intact.

Finally let's turn to the last case $($ iii), where the inhomogeneities are constrained by the conditions (4.10) and (4.11). For this purpose we need to consider the similarity transformation in the quantum space that interchanges the two groups of inhomogeneities for the monodromy matrix, while preserving their ordering inside of each group

$$
\boldsymbol{M}\left(\zeta \mid \eta_{N / 2}, \ldots, \eta_{1}, \eta_{N}, \ldots, \eta_{N / 2+1}\right)=\hat{\mathrm{S}}_{3}^{-1} \boldsymbol{M}\left(\zeta \mid \eta_{N}, \ldots, \eta_{N / 2+1}, \eta_{N}, \ldots, \eta_{1}\right) \hat{\mathrm{S}}_{3}
$$

One can show that

$$
\hat{\mathrm{S}}_{3}=\prod_{m=N / 2+1}^{\curvearrowleft}\left[\prod_{n=0}^{\curvearrowleft} \check{\boldsymbol{R}}_{m-n, m-n-1}\left(\eta_{N-n} / \eta_{m-N / 2}\right)\right] .
$$

Introduce the matrix $\hat{U}$ as

$$
\eta_{J}=\Lambda^{-1} \omega_{J}, \quad \eta_{J+N / 2}=\Lambda \omega_{J}, \quad\left|\omega_{J}\right|=1: \quad \hat{\mathrm{U}}=\hat{\mathrm{S}}_{3} \prod_{J=1}^{N} \sigma_{J}^{x} .
$$

It turns out that it also satisfies (4.13) and, for the time-reversal conjugation (4.12) with $\hat{U}$ defined as above, equations (4.15) and (4.16) continue to hold.

This way in all of the cases $(i)-(i i i)$ of the reality conditions imposed on the inhomogeneities, it is possible to introduce the anti-unitary operator (4.12) such that equations (4.15) and (4.16) are satisfied. The matrix $\hat{U}$ takes the general form

$$
\hat{\mathrm{U}}=\hat{\mathrm{S}}_{\mathcal{T}} \prod_{J=1}^{N} \sigma_{J}^{x}
$$

where $\hat{S}_{\mathcal{T}}$ depends on the particular case being considered. Namely when the inhomogeneities are constrained by (4.8), $\hat{\mathrm{S}}_{\mathcal{T}}$ is the identity operator. For the cases (4.9) and (4.10) $\hat{\mathrm{S}}_{\mathcal{T}}$ is given by $\hat{\mathrm{S}}_{2}(4.20)$ and $\hat{\mathrm{S}}_{3}(4.21)$, respectively. 


\section{$5 \quad$ Hermitian structure}

There are many ways to introduce the Hermitian structure, i.e., the sesquilinear form for the states and the Hermitian conjugation of operators in the $2^{N}$ dimensional linear space $\mathscr{V}_{N}(2.3)$. Here we discuss the Hermitian structures that are consistent with the integrable structure of the model.

Consider each factor in the product in the r.h.s. of equation (2.13). Under the standard matrix ("dagger") conjugation in the quantum space $\mathscr{V}_{N}$, they transform as

$$
\left[q^{-\frac{1}{2}} \boldsymbol{R}_{m}\left(q \zeta / \eta_{m}\right)\right]^{\dagger}=\sigma^{x}\left(\zeta^{*} / \eta_{m}^{*}\right)^{-\frac{1}{2} \sigma^{z}}\left[q^{-\frac{1}{2}} \boldsymbol{R}_{m}\left(q \zeta^{*} / \eta_{m}^{*}\right)\right]\left(\zeta^{*} / \eta_{m}^{*}\right)^{+\frac{1}{2} \sigma^{z}} \sigma^{x}
$$

In turn, taking the $\dagger$-conjugation of the monodromy matrix results in

$$
\left[\boldsymbol{M}\left(\zeta \mid \eta_{N}, \eta_{N-1}, \ldots, \eta_{1}\right)\right]^{\dagger}=\sigma^{x}\left(\zeta^{*}\right)^{-\frac{1}{2} \sigma^{z}} \hat{\mathrm{V}} \boldsymbol{M}\left(\zeta^{*} \mid \eta_{N}^{*}, \eta_{N-1}^{*}, \ldots, \eta_{1}^{*}\right) \hat{\mathrm{V}}^{-1}\left(\zeta^{*}\right)^{+\frac{1}{2} \sigma^{z}} \sigma^{x}
$$

where

$$
\hat{\mathrm{V}}=\prod_{J=1}^{N}\left(\eta_{J}^{*}\right)^{-\frac{1}{2} \sigma_{J}^{z}} .
$$

Now suppose that the (non-ordered) set of inhomogeneities coincides with the complex conjugated set:

$$
\left\{\eta_{J}^{*}\right\}_{J=1}^{N}=\left\{\eta_{J}\right\}_{J=1}^{N}
$$

In particular, this property holds for all the three cases (4.8), (4.9) and (4.10) considered above. Then there exists a similarity transformation such that

$$
\boldsymbol{M}\left(\zeta \mid \eta_{N}, \eta_{N-1}, \ldots, \eta_{1}\right)=\hat{\mathrm{S}}^{-1} \boldsymbol{M}\left(\zeta \mid \eta_{N}^{*}, \eta_{N-1}^{*}, \ldots, \eta_{1}^{*}\right) \hat{\mathrm{S}}, \quad \hat{\mathrm{S}} \in \operatorname{End}\left(\mathscr{V}_{N}\right)
$$

Combining the latter with equation (5.1), yields

$$
[\boldsymbol{M}(\zeta)]^{\dagger}=\sigma^{x}\left(\zeta^{*}\right)^{-\frac{1}{2} \sigma^{z}}\left[\hat{\mathrm{X}} \boldsymbol{M}\left(\zeta^{*}\right) \hat{\mathrm{X}}^{-1}\right]\left(\zeta^{*}\right)^{+\frac{1}{2} \sigma^{z}} \sigma^{x}
$$

with the Hermitian matrix $\hat{\mathrm{X}}$ given by

$$
\hat{\mathrm{X}}=\hat{\mathrm{X}}^{\dagger}=\hat{\mathrm{V}} \hat{\mathrm{S}}^{-1}, \quad \hat{\mathrm{X}} \in \operatorname{End}\left(\mathscr{V}_{N}\right) .
$$

Formula (5.4) implies that, in general, the transfer matrix is not Hermitian w.r.t. the $†$-conjugation. However, for an arbitrary $\hat{O} \in \operatorname{End}\left(\mathscr{V}_{N}\right)$, one can introduce the non-standard conjugation as

$$
\hat{\mathrm{O}}^{\ddagger}=\hat{\mathrm{X}}^{-1} \hat{\mathrm{O}}^{\dagger} \hat{\mathrm{X}} \text {. }
$$

Since the matrix $\hat{X}$ is Hermitian (5.5), it is guaranteed that $\left(\hat{O}^{\ddagger}\right)^{\ddagger}=\hat{O}$ so that (5.6) indeed defines a conjugation. Then equation (5.4) can be rewritten as the $\ddagger$-conjugation condition for the operator valued entries of the monodromy matrix (2.15):

$$
\begin{array}{ll}
{[\hat{\mathrm{A}}(\zeta)]^{\ddagger}=\hat{\mathrm{D}}\left(\zeta^{*}\right),} & {[\hat{\mathrm{D}}(\zeta)]^{\ddagger}=\hat{\mathrm{A}}\left(\zeta^{*}\right),} \\
{[\hat{\mathrm{B}}(\zeta)]^{\ddagger}=\zeta^{*} \hat{\mathrm{C}}\left(\zeta^{*}\right),} & {[\hat{\mathrm{C}}(\zeta)]^{\ddagger}=\left(\zeta^{*}\right)^{-1} \hat{\mathrm{B}}\left(\zeta^{*}\right) .}
\end{array}
$$

In turn, these relations imply

$$
[\mathbb{T}(\zeta)]^{\ddagger}=\mathbb{T}\left(\zeta^{*}\right) .
$$


In a similar way, one can show that

$$
\left[\mathbb{A}_{ \pm}(\zeta)\right]^{\ddagger}=\mathbb{A}_{ \pm}\left(\zeta^{*}\right) .
$$

As a matter of fact, there is a more general version of (5.6) for which the condition similar to (5.8) holds. Indeed consider the conjugation of the form

$$
\hat{\mathrm{O}}^{\star}=\mathbb{Y}^{-1} \hat{\mathrm{O}}^{\ddagger} \mathbb{Y}=\mathbb{Y}^{-1} \hat{\mathrm{X}}^{-1} \hat{\mathrm{O}}^{\dagger} \hat{\mathrm{X}} \mathbb{Y} .
$$

In order to fulfill the requirement $\left(\hat{\mathrm{O}}^{\star}\right)^{\star}=\hat{\mathrm{O}}$, the operator $\mathbb{Y}$ should be such that

$$
\mathbb{Y}^{\ddagger}=\mathbb{Y}
$$

If, in addition,

$$
\left[\mathbb{Y}, \mathbb{A}_{ \pm}(\zeta)\right]=0
$$

then it follows from (5.8) that

$$
[\mathbb{T}(\zeta)]^{\star}=\mathbb{T}\left(\zeta^{*}\right), \quad\left[\mathbb{A}_{ \pm}(\zeta)\right]^{\star}=\mathbb{A}_{ \pm}\left(\zeta^{*}\right)
$$

The $\ddagger$-conjugation is a special case of the $\star$-conjugation with $\mathbb{Y}=\hat{\mathbf{1}}$.

For a given $\mathbb{Y} \in \operatorname{End}\left(\mathscr{V}_{N}\right)$ satisfying (5.10) and (5.11) there is a unique sesquilinear form $\left(\boldsymbol{\psi}_{2}, \boldsymbol{\psi}_{1}\right)_{\star}\left(\boldsymbol{\psi}_{1,2} \in \mathscr{V}_{N}\right)$ that is compatible with the $\star$-conjugation (5.9). It is defined by the requirement

$$
\left(\boldsymbol{\psi}_{2}, \hat{\mathrm{O}} \boldsymbol{\psi}_{1}\right)_{\star}=\left(\hat{\mathrm{O}}^{\star} \boldsymbol{\psi}_{2}, \boldsymbol{\psi}_{1}\right)_{\star}
$$

and the overall normalization condition for the pseudovacuum $\boldsymbol{\Psi}_{0} \equiv|0\rangle(2.6)$ :

$$
\left(\mathbf{\Psi}_{0}, \mathbf{\Psi}_{0}\right)_{\star}=1
$$

The second equality in (5.12), together with the fact that the spectrum of the operators $\mathbb{A}_{ \pm}(\zeta)$ is expected to be non-degenerate for generic values of $\mathrm{k}$, implies that the Bethe states (2.18) satisfy an orthogonality condition of the form

$$
\left(\Psi^{\prime}, \Psi\right)_{\star}=0 \quad \text { unless } \quad \Psi^{\prime}=\hat{\mathcal{C}} \hat{\mathcal{P}} \hat{\mathcal{T}} \boldsymbol{\Psi} .
$$

Let us define the "norm" for an arbitrary state $\boldsymbol{\psi} \in \mathscr{V}_{N}$ as

$$
\mathfrak{N}_{Y}[\boldsymbol{\psi}]:=(\hat{\mathcal{C}} \hat{\mathcal{P}} \hat{\mathcal{T}} \boldsymbol{\psi}, \boldsymbol{\psi})_{\star} .
$$

For the Bethe state corresponding to the set of Bethe roots $\left\{\zeta_{m}\right\}_{m=1}^{M}$, taking into account equations (5.7) and (2.19), its norm is given by

$$
\mathfrak{N}_{Y}[\boldsymbol{\Psi}]=Y\left(\zeta_{1}, \ldots, \zeta_{M}\right) \mathfrak{K}[\boldsymbol{\Psi}] \prod_{m=1}^{M} \zeta_{m}
$$

where $Y\left(\zeta_{1}, \ldots, \zeta_{M}\right)$ stands for the eigenvalue of the operator $\mathbb{Y}$ on the Bethe state $\boldsymbol{\Psi}$.

One can construct a variety of matrices $\mathbb{Y}$ obeying equations (5.10) and (5.11). For example, taking into account (5.8a), it is easy to see that both conditions are satisfied for $\mathbb{Y}=$ $\mathrm{e}^{\mathrm{i} \pi\left(\mathbb{S}^{z}-\frac{N}{2}\right)} \mathbb{A}_{+}^{(\infty)}$, where $\mathbb{A}_{+}^{(\infty)}$ is defined through the formula (3.12). In this case the eigenvalues of $\mathbb{Y}$ are given by the product $\prod_{m=1}^{M} \zeta_{m}^{-1}$, so that the norm (5.13) coincides with $\mathfrak{K}[\mathbf{\Psi}]$. In other 
words, the functional formally introduced in (2.20) may be interpreted as a particular norm of the Bethe state

$$
\mathfrak{K}[\Psi]=\mathfrak{N}_{Y}[\boldsymbol{\Psi}] \quad \text { with } \quad \mathbb{Y}=\mathrm{e}^{\mathrm{i} \pi\left(\mathbb{S}^{z}-\frac{N}{2}\right)} \mathbb{A}_{+}^{(\infty)} .
$$

It should be emphasized that different operators $\mathbb{Y}$ may lead to equivalent Hermitian structures. Replacing $\mathbb{Y}$ by $\mathbb{Y}^{\prime}$ such that

$$
Y^{\prime}\left(\zeta_{1}, \ldots, \zeta_{M}\right)=\alpha\left(\zeta_{1}, \ldots, \zeta_{M}\right)\left(\alpha\left(\zeta_{1}^{*}, \ldots, \zeta_{M}^{*}\right)\right)^{*} Y\left(\zeta_{1}, \ldots, \zeta_{M}\right)
$$

with $\left(\alpha\left(\zeta_{1}, \ldots, \zeta_{M}\right)\right)^{ \pm 1} \neq 0$ for any Bethe state, may be compensated for by a change of the normalization

$$
\Psi \mapsto \alpha\left(\zeta_{1}, \ldots, \zeta_{M}\right) \Psi .
$$

However, though the Hermitian structures corresponding to $\mathbb{Y}$ and $\mathbb{Y}^{\prime}(5.15)$ are equivalent in the formal linear algebra sense, the action of the conjugation (5.9) on the local spin operators could be radically different. In particular, a conjugation that acts locally on $\sigma_{J}^{A}$ may become highly non-local upon the substitution $\mathbb{Y} \mapsto \mathbb{Y}^{\prime}$.

The above discussion is valid for any set of inhomogeneities provided that (5.2) is satisfied. However, the Hermitian matrix $\hat{X}$ (5.5), which was left unspecified, depends on the precise form of the reality conditions imposed on $\left\{\eta_{J}\right\}$. For the three cases from the previous section one has:

(i) For unimodular inhomogeneities $\eta_{J}^{*}=\eta_{J}^{-1}=\eta_{N+1-J}$, the matrix $\hat{\mathrm{S}}$ (5.3) coincides with $\hat{\mathrm{S}}_{2}$ from (4.20). Hence $\hat{X}$ is given by

$$
\eta_{J}^{*}=\eta_{J}^{-1}=\eta_{N+1-J}: \quad \hat{\mathrm{X}}=\left(\prod_{J=1}^{N}\left(\eta_{J}\right)^{\frac{1}{2} \sigma_{J}^{z}}\right) \hat{\mathrm{S}}_{2}^{-1} .
$$

(ii) In the case with $\eta_{J}$ real (4.9), the matrix $\hat{\mathrm{S}}$ is the identity and $\hat{\mathrm{X}}$ reads as

$$
\eta_{J}^{*}=\eta_{J}=\left(\eta_{N+1-J}\right)^{-1}: \quad \hat{\mathrm{X}}=\prod_{J=1}^{N}\left(\eta_{J}\right)^{-\frac{1}{2} \sigma_{J}^{z}} .
$$

(iii) For the case (4.10), where the number of columns $N$ is assumed to be even, the ordered set of inhomogeneities satisfies the condition

$$
\left(\eta_{N}^{*}, \ldots, \eta_{N / 2+1}^{*}, \eta_{N / 2}^{*}, \ldots, \eta_{1}^{*}\right)=\left(\eta_{N / 2+1}, \ldots, \eta_{N}, \eta_{1}, \ldots, \eta_{N / 2}\right) .
$$

It is convenient to write $\hat{S}$ from (5.3) as the product $\hat{S}=\hat{S}_{I} \hat{S}_{I I}$ and the matrices $\hat{S}_{I}$ and $\hat{S}_{I I}$ reverse the order of the inhomogeneities of $\left\{\eta_{J}\right\}_{J=1}^{N / 2}$ and $\left\{\eta_{N / 2+J}\right\}_{J=1}^{N / 2}$ respectively, i.e.,

$$
\begin{aligned}
& \boldsymbol{M}\left(\zeta \mid \eta_{N}, \ldots, \eta_{N / 2+1}, \eta_{N / 2}, \ldots, \eta_{1}\right)=\hat{\mathrm{S}}_{\mathrm{I}}^{-1} \boldsymbol{M}\left(\zeta \mid \eta_{N}, \ldots, \eta_{N / 2+1}, \eta_{1}, \ldots, \eta_{N / 2}\right) \hat{\mathrm{S}}_{\mathrm{I}} \\
& \boldsymbol{M}\left(\zeta \mid \eta_{N}, \ldots, \eta_{N / 2+1}, \eta_{N / 2}, \ldots, \eta_{1}\right)=\hat{\mathrm{S}}_{\mathrm{II}}^{-1} \boldsymbol{M}\left(\zeta \mid \eta_{N / 2+1}, \ldots, \eta_{N}, \eta_{N / 2}, \ldots, \eta_{1}\right) \hat{\mathrm{S}}_{\mathrm{II}} .
\end{aligned}
$$

The expression for these matrices are obtained from equation (4.20) by a simple replacement of the indices

$$
\begin{aligned}
\hat{\mathrm{S}}_{\mathrm{I}} & =\prod_{m=2}^{\curvearrowleft / 2}\left[\prod_{n=N / 2-m+1}^{\curvearrowleft} \check{\boldsymbol{R}}_{n+1, n}\left(\eta_{m} / \eta_{n+m-N / 2}\right)\right], \\
\hat{\mathrm{S}}_{\mathrm{II}} & =\prod_{m=2}^{N / 2-1}\left[\prod_{n=N-m+1}^{\curvearrowleft / 1} \check{\boldsymbol{R}}_{n+1, n}\left(\eta_{m+N / 2} / \eta_{n+m-N / 2}\right)\right] .
\end{aligned}
$$


Then the matrix $\hat{X}$ is given by

$$
\begin{aligned}
& \eta_{J}=\Lambda^{-1} \omega_{J}, \quad \eta_{L+J}=\Lambda \omega_{J} \\
& \left|\omega_{J}\right|=1: \quad \hat{\mathrm{X}}=\left(\prod_{J=1}^{N / 2}\left(\Lambda^{+1} \omega_{J}\right)^{+\frac{1}{2} \sigma_{J}^{z}}\left(\Lambda^{-1} \omega_{J}\right)^{+\frac{1}{2} \sigma_{J+N / 2}^{z}}\right)\left(\hat{\mathrm{S}}_{\mathrm{I}} \hat{\mathrm{S}}_{\mathrm{II}}\right)^{-1} .
\end{aligned}
$$

\section{Lattice translation symmetry}

Translational invariance is a fundamental symmetry of a local quantum field theory. Having in mind the study of the universality classes for the inhomogeneous six-vertex model, it is natural to focus on the lattice, where the number of columns $N$ is divisible by some integer $r$,

$$
N=r L,
$$

while the inhomogeneities satisfy the $r$-site periodicity conditions

$$
\eta_{J+r}=\eta_{J} \quad(J=1,2, \ldots, N)
$$

(here by definition we take $\eta_{J+N} \equiv \eta_{J}$ ). With these restrictions, the commuting family contains a set of operators that play a central rôle for the description of the critical behaviour. The aim of this section is to introduce these operators and discuss their basic properties.

\section{$6.1 \quad r$-site translation operator}

The one-site translation operator may be defined without any restrictions imposed on the parameters of the model. Taking into account the quasi-periodic boundary conditions with twist parameter $\omega=\mathrm{e}^{\mathrm{i} \pi \mathrm{k}}$, its matrix elements are given by

$$
(\hat{\mathcal{K}})_{a_{N} a_{N-1} \cdots a_{1}}^{b_{N} b_{N-1} \cdots b_{1}}=\mathrm{e}^{\mathrm{i} \pi \mathrm{k} a_{1}} \delta_{a_{1}}^{b_{N}} \delta_{a_{N}}^{b_{N-1}} \cdots \delta_{a_{2}}^{b_{1}} .
$$

The action of the one-site translation on the local spin operators reads as

$$
\begin{aligned}
& \hat{\mathcal{K}} \sigma_{J}^{A} \hat{\mathcal{K}}^{-1}=\sigma_{J+1}^{A} \quad(A=x, y, z ; J=1, \ldots, N-1), \\
& \hat{\mathcal{K}} \sigma_{N}^{A} \hat{\mathcal{K}}^{-1}=\mathrm{e}^{+\mathrm{i} \pi \mathrm{k} \sigma_{1}^{z}} \sigma_{1}^{A} \mathrm{e}^{-\mathrm{i} \pi \mathrm{k} \sigma_{1}^{z}} .
\end{aligned}
$$

In turn, the adjoint action of $\mathcal{K}$ on the transfer matrix (2.17) results in a cyclic permutation of the inhomogeneities:

$$
\hat{\mathcal{K}} \mathbb{T}\left(\zeta \mid \eta_{N}, \eta_{N-1}, \ldots, \eta_{1}\right) \hat{\mathcal{K}}^{-1}=\mathbb{T}\left(\zeta \mid \eta_{N-1}, \eta_{N-2}, \ldots, \eta_{N}\right) .
$$

This relation shows that with the conditions (6.1) and (6.2) imposed, the $r$-site translation operator belongs to the commuting family,

$$
\mathbb{K}=\hat{\mathcal{K}}^{r}: \quad \mathbb{K}^{L}=\mathrm{e}^{2 \pi \mathrm{ikS} \mathbb{S}^{z}}, \quad[\mathbb{K}, \mathbb{T}(\zeta)]=0 .
$$

In fact $\mathbb{K}$ not only commutes, but it can be expressed in terms of the transfer matrix. Assuming the normalization $(2.11)$ for $\mathbb{T}(\zeta)$ and taking into account (2.12), it's straightforward to show

$$
\mathbb{K}=\left(q-q^{-1}\right)^{-N}\left[\prod_{1<m<\ell<r}\left(q^{2}+q^{-2}-\frac{\eta_{\ell}}{\eta_{m}}-\frac{\eta_{m}}{\eta_{\ell}}\right)^{-L}\right] \prod_{\ell=1}^{r} q^{\frac{N}{2}} \mathbb{T}\left(-q^{-1} \eta_{\ell}\right) .
$$

It follows that the eigenvalue of the $r$-site translation operator on the Bethe state (2.18) is given by

$$
\mathbb{K} \boldsymbol{\Psi}=K \boldsymbol{\Psi}: \quad K=\mathrm{e}^{\mathrm{i} \pi r \mathrm{k}} q^{r S^{z}-\frac{r N}{2}} \prod_{\ell=1}^{r} \frac{A_{+}\left(-q^{+1} \eta_{\ell}\right)}{A_{+}\left(-q^{-1} \eta_{\ell}\right)},
$$

where $A_{+}(\zeta)$ is the same as in (3.1). 


\subsection{Quasi-shift operators}

In [25], which was devoted to the study of the $\mathcal{Z}_{2}$ invariant model, the authors introduced the so-called quasi-shift operator. It turns out to be a key player for the description of the scaling limit. Similar operators may be defined for the general model provided the restrictions (6.1) and (6.2) are imposed. They are likewise expected to be important for analyzing the critical behaviour.

The construction of the quasi-shift operators is based on the following observation. When the restrictions (6.2) on the $\eta_{J}$ are imposed, the cyclic permutation of the inhomogeneities within each group $\left(\eta_{J+r}, \eta_{J+r-1}, \ldots, \eta_{J+1}\right) \mapsto\left(\eta_{J+1}, \eta_{J+r}, \ldots, \eta_{J+2}\right)$ with $J=\ell(\bmod r)$ is equivalent to an overall cyclic permutation of the ordered set $\left(\eta_{N}, \eta_{N-1}, \ldots, \eta_{1}\right)$. In view of equation (4.18), this implies that there exists $r$ inequivalent operators $\hat{\mathcal{D}}^{(\ell)}$, such that

$$
\left(\hat{\mathcal{D}}^{(\ell)}\right)^{-1} \mathbb{T}\left(\zeta \mid \eta_{N}, \eta_{N-1}, \ldots, \eta_{1}\right) \hat{\mathcal{D}}^{(\ell)}=\mathbb{T}\left(\zeta \mid \eta_{N-1}, \eta_{N-2}, \ldots, \eta_{N}\right), \quad \ell=1,2, \ldots, r .
$$

Explicitly, they are given by

$$
\hat{\mathcal{D}}^{(\ell)}=\prod_{j=0}^{L-1}\left(\hat{\mathcal{K}}^{\ell+r j}\left[\prod_{m=1}^{r-1} \check{\boldsymbol{R}}_{m+1, m}\left(\eta_{\ell} / \eta_{\ell+m}\right)\right] \hat{\mathcal{K}}^{-\ell-r j}\right),
$$

where the outer product is unordered since the factors inside it commute with each other. It is worth mentioning that the operator

$$
\hat{\mathcal{D}}^{(r)}=\prod_{m=1}^{\curvearrowleft-1} \check{\boldsymbol{R}}_{m+1, m}\left(\eta_{r} / \eta_{m}\right)
$$

plays a special rôle. Though the adjoint action of $\hat{\mathcal{D}}^{(\ell)}$ on the transfer matrix results in a permutation of the inhomogeneities as in (6.6), a similar formula does not hold for the entries $\hat{A}$, $\hat{B}, \hat{C}$ and $\hat{D}$ of the monodromy matrix. However, it turns out that

$$
\left(\hat{\mathcal{D}}^{(r)}\right)^{-1} \boldsymbol{M}\left(\zeta \mid \eta_{N}, \eta_{N-1}, \ldots, \eta_{1}\right) \hat{\mathcal{D}}^{(r)}=\boldsymbol{M}\left(\zeta \mid \eta_{N-1}, \eta_{N-2}, \ldots, \eta_{N}\right) .
$$

Combining equations (6.4) and (6.6) it is easy to see that the operators

$$
\mathbb{K}^{(\ell)}=\hat{\mathcal{D}}^{(\ell)} \hat{\mathcal{K}}, \quad \ell=1,2, \ldots, r,
$$

belong to the commuting family,

$$
\left[\mathbb{K}^{(\ell)}, \mathbb{T}(\zeta)\right]=0
$$

Moreover, their product coincides with the $r$-site translation:

$$
\prod_{\ell=1}^{r} \mathbb{K}^{(\ell)}=\mathbb{K}
$$

We will refer to $\mathbb{K}^{(\ell)}$ as the quasi-shift operators. ${ }^{5}$ Similar as in equation (6.5), it is possible to express them in terms of the transfer matrix as

$$
\mathbb{K}^{(\ell)}=q^{-\frac{N}{2}}\left(\prod_{J=1}^{N}\left(\eta_{J}-q^{-2} \eta_{\ell}\right)^{-1}\right) \mathbb{T}\left(-q^{-1} \eta_{\ell}\right) .
$$

\footnotetext{
${ }^{5}$ There is a slight difference between the terminology used here and that from the work [25]. The latter focuses on the $\mathcal{Z}_{2}$ invariant model, where there are two operators $\mathbb{K}^{(1)}$ and $\mathbb{K}^{(2)}$ such that $\mathbb{K}^{(1)} \mathbb{K}^{(2)}=\mathbb{K}$. They introduce the "quasi-momentum", which essentially coincides with the logarithm of $\mathbb{K}^{(1)}\left(\mathbb{K}^{(2)}\right)^{-1}$. The term "quasi-shift" was coined in [21].
} 
Their eigenvalues for the Bethe state (2.18) are given by

$$
K^{(\ell)}=\mathrm{e}^{\mathrm{i} \pi \mathrm{k}} q^{S^{z}-N / 2} \frac{A_{+}\left(-q^{+1} \eta_{\ell}\right)}{A_{+}\left(-q^{-1} \eta_{\ell}\right)}
$$

with $A_{+}(\zeta)$ from (3.1).

\subsection{Hamiltonians}

In the case of the homogeneous six-vertex model the transfer matrix commutes with the spin $\frac{1}{2}$ Heisenberg $X X Z$ Hamiltonian. A similar important property holds true for the model, where the parameters $\eta_{J}$ satisfy the periodicity conditions (6.2) with any $r \geq 1$. Namely the corresponding commuting family contains spin chain Hamiltonians that are given by a sum of terms, each of which is built out of local spin operators from $r+1$ consecutive sites of the lattice. There are $r$ such Hamiltonians and, in terms of the row-to-row transfer matrix, they are expressed as

$$
\mathbb{H}^{(\ell)}=\left.2 \mathrm{i} \zeta \partial_{\zeta} \log \left(\mathbb{T}\left(-q^{-1} \zeta\right)\right)\right|_{\zeta=\eta_{\ell}}-2 \mathrm{i} \sum_{J=1}^{N}\left(1-q^{2} \eta_{J} / \eta_{\ell}\right)^{-1}, \quad \ell=1,2, \ldots, r
$$

A calculation shows that

$$
\mathbb{H}^{(\ell)}=\sum_{m=0}^{L-1} \mathbb{K}^{m} \hat{\mathbf{H}}^{(\ell)} \mathbb{K}^{-m}
$$

where $\hat{\mathbf{H}}^{(\ell)} \in \operatorname{End}\left(\mathscr{V}_{N}\right)$ are built from the $\sigma_{J}^{A}$ with $J=\ell, \ell+1, \ldots, \ell+r$. Assuming that $L \geq 2$ so that the sites $\ell$ and $\ell+r$ are not identified with one another, the operators $\hat{\mathrm{H}}^{(\ell)}$ are given by

$$
\hat{\mathrm{H}}^{(\ell)}=\sum_{m=1}^{r} \hat{\mathrm{S}}_{m}^{(\ell)} \hat{\mathrm{J}}_{\ell+m, \ell+m-1}\left(\eta_{\ell} / \eta_{\ell+m-1}\right)\left(\hat{\mathrm{S}}_{m}^{(\ell)}\right)^{-1} \text {. }
$$

Here we use the notation

$$
\hat{\mathrm{J}}_{n+1, n}(\zeta)=2 \mathrm{i} \zeta \partial_{\zeta} \log \left(\check{\boldsymbol{R}}_{n+1, n}(\zeta)\right)
$$

and

$$
\hat{\mathrm{S}}_{m}^{(\ell)}=\prod_{n=\ell+m}^{\curvearrowleft} \varlimsup_{\substack{\ell+r-1 \\ n+1, n}}\left(\eta_{\ell} / \eta_{n}\right), \quad \hat{\mathrm{S}}_{r}^{(\ell)}=\hat{\mathbf{1}}
$$

with $\check{\boldsymbol{R}}_{n+1, n}$ as in (4.19).

\subsection{Interplay with $\mathcal{C P}, \mathcal{T}$ and Hermitian conjugation}

In order to incorporate the $\mathcal{C}, \mathcal{P}$ and $\mathcal{T}$ conjugations for the model with translational invariance, extra constraints need to be imposed on the parameters in addition to (6.2). For the three cases considered in Section 4, a brief examination shows that the first and second ones (4.8), (4.9) are compatible with the periodicity condition $\eta_{J+r}=\eta_{J}$ while the last case (4.10) is not. Assuming the inhomogeneities are as in $(i)$ or $(i i)$, the action of $\mathcal{C P}$ and $\mathcal{T}$ on the quasi-shift operators and the Hamiltonians is given by

$$
\hat{\mathcal{C}} \hat{\mathcal{P}} \mathbb{H}^{(\ell)} \hat{\mathcal{C}} \hat{\mathcal{P}}=\mathbb{H}^{(r-\ell+1)}, \quad \hat{\mathcal{C}} \hat{\mathcal{P}} \mathbb{K}^{(\ell)} \hat{\mathcal{C}} \hat{\mathcal{P}}=\left(\mathbb{K}^{(r-\ell+1)}\right)^{-1}
$$


and

$$
\begin{array}{ll}
\text { case }(i): & \hat{\mathcal{T}} \mathbb{H}^{(\ell)} \hat{\mathcal{T}}=\mathbb{H}^{(\ell)}, \quad \hat{\mathcal{T}} \mathbb{K}^{(\ell)} \hat{\mathcal{T}}=\mathbb{K}^{(\ell)}, \\
\text { case }(i i): & \hat{\mathcal{T}} \mathbb{H}^{(\ell)} \hat{\mathcal{T}}=\mathbb{H}^{(r-\ell+1)}, \quad \hat{\mathcal{T}} \mathbb{K}^{(\ell)} \hat{\mathcal{T}}=\mathbb{K}^{(r-\ell+1)} .
\end{array}
$$

These follow from formulae (6.10) and (6.11), which express $\mathbb{K}^{(\ell)}$ and $\mathbb{H}^{(\ell)}$ in terms of $\mathbb{T}(\zeta)$, and equations (4.7), (4.15) that describe the commutation relations of $\hat{\mathcal{C}} \hat{\mathcal{P}}$ and $\hat{\mathcal{T}}$ with the transfer matrix.

The Hermitian conjugation (5.9) that is consistent with the integrable structure involves the matrix $\hat{X}$, which depends on the reality conditions imposed on the set $\left\{\eta_{J}^{*}\right\}_{J=1}^{N}=\left\{\eta_{J}\right\}_{J=1}^{N}$. In the case $(i)$, the expressions (5.16), (4.20) can be simplified if the $r$-site periodicity conditions (6.2) are taken into account. It turns out that

$$
\hat{\mathrm{X}}=\prod_{m=0}^{L-1} \mathbb{K}^{m} \hat{\mathrm{X}}^{(1)} \mathbb{K}^{-m}, \quad \hat{\mathrm{X}}^{(1)}=\left(\prod_{\ell=1}^{r}\left(\eta_{\ell}\right)^{\frac{1}{2} \sigma_{\ell}^{z}}\right)\left(\hat{\mathrm{S}}_{2}^{(1)}\right)^{-1},
$$

where

$$
\hat{\mathrm{S}}_{2}^{(1)}=\prod_{m=2}^{\curvearrowleft}\left[\prod_{n=r-m+1}^{\curvearrowleft} \check{\boldsymbol{R}}_{n+1, n}\left(\eta_{m} / \eta_{n+m-r}\right)\right] .
$$

Notice that each of the terms $\mathbb{K}^{m} \hat{\mathbf{X}}^{(1)} \mathbb{K}^{-m}$ entering into the product expression (6.12) acts nontrivially only on sites $m r+1, m r+2, \ldots, m r+r$. For case $(i i)$ from Section $4, \hat{\mathrm{X}}$ is the diagonal matrix given by equation (5.17).

The formula for the $\star$-conjugation also contains the operator $\mathbb{Y}$, subject to the conditions (5.10) and (5.11). Independently of its choice $(\mathbb{T}(\zeta))^{\star}=\mathbb{T}\left(\zeta^{*}\right)$ from which it follows that

$$
\begin{array}{ll}
\text { case }(i): & \left(\mathbb{H}^{(\ell)}\right)^{\star}=\mathbb{H}^{(r-\ell+1)}, \quad\left(\mathbb{K}^{(\ell)}\right)^{\star}=\left(\mathbb{K}^{(r-\ell+1)}\right)^{-1}, \\
\text { case }(i i): & \left(\mathbb{H}^{(\ell)}\right)^{\star}=\mathbb{H}^{(\ell)}, \quad\left(\mathbb{K}^{(\ell)}\right)^{\star}=\left(\mathbb{K}^{(\ell)}\right)^{-1} .
\end{array}
$$

Though the Hamiltonians and quasi-shift operators are not preserved under the $\mathcal{C P}$ transformation and the $\star$-conjugation, the combinations

$$
\mathbb{H}=\sum_{\ell=1}^{r} \mathbb{H}^{(\ell)}, \quad \mathbb{K}=\prod_{\ell=1}^{r} \mathbb{K}^{(\ell)}
$$

satisfy the conditions

$$
\begin{array}{lrl}
\hat{\mathcal{C}} \hat{\mathcal{P}} \mathbb{H} \hat{\mathcal{C}} \hat{\mathcal{P}}=\mathbb{H}, & \hat{\mathcal{T}} \mathbb{H} \hat{\mathcal{T}}=\mathbb{H}, & \mathbb{H}^{\star}=\mathbb{H}, \\
\hat{\mathcal{C}} \hat{\mathcal{P}} \mathbb{K} \hat{\mathcal{C}} \hat{\mathcal{P}}=\mathbb{K}^{-1}, & \hat{\mathcal{T}} \mathbb{K} \hat{\mathcal{T}}=\mathbb{K}, & \mathbb{K}^{\star}=\mathbb{K}^{-1}
\end{array}
$$

for both cases $(i)$ and $(i i)$.

\section{$7 \quad \mathcal{Z}_{r}$-invariance}

The operators $\hat{\mathcal{D}}^{(\ell)} \in \operatorname{End}\left(\mathscr{V}_{N}\right)$, acting on the transfer matrix, result in a cyclic shift of the inhomogeneities as in equation (6.6). Taking into account that $\eta_{J+r}=\eta_{J}$, after $r$ consecutive applications of this transformation the inhomogeneities will return to their original order. Thus $\left(\hat{\mathcal{D}}^{(\ell)}\right)^{r}$ commutes with $\mathbb{T}(\zeta)$. It turns out that if the inhomogeneities are specified to be

$$
\eta_{J}=(-1)^{r} \mathrm{e}^{\frac{\mathrm{i} \pi}{r}(2 J-1)},
$$


then further

$$
\left(\hat{\mathcal{D}}^{(\ell)}\right)^{r}=\hat{\mathbf{1}}, \quad \ell=1,2, \ldots, r .
$$

In this case all the operators $\hat{\mathcal{D}}^{(\ell)}$ can be expressed in terms of

$$
\hat{\mathcal{D}} \equiv \hat{\mathcal{D}}^{(r)}=\prod_{m=1}^{\curvearrowleft} \check{\boldsymbol{R}}_{m+1, m}\left(\mathrm{e}^{-2 \mathrm{i} \pi m / r}\right)
$$

and the one-site translation operator $\mathcal{K}(6.3)$. Namely,

$$
\hat{\mathcal{D}}^{(\ell)}=\hat{\mathcal{K}}^{\ell} \hat{\mathcal{D}} \hat{\mathcal{K}}^{-\ell}
$$

with

$$
\hat{\mathcal{K}}^{r}=\mathbb{K}, \quad \hat{\mathcal{D}}^{r}=1 .
$$

When the condition (7.1) is imposed, both operators $\hat{\mathcal{D}}$ and $\hat{\mathcal{K}}$ preserve the commuting family. Their adjoint action on the transfer matrix and $\mathbb{A}_{ \pm}(\zeta)$ is given by the similar formulae

$$
\begin{array}{ll}
\hat{\mathcal{D}}^{-1} \mathbb{T}(\zeta) \hat{\mathcal{D}}=\mathbb{T}\left(\mathrm{e}^{+2 \pi \mathrm{i} / r} \zeta\right), & \hat{\mathcal{D}}^{-1} \mathbb{A}_{ \pm}(\zeta) \hat{\mathcal{D}}=\mathbb{A}_{ \pm}\left(\mathrm{e}^{+2 \pi \mathrm{i} / r} \zeta\right), \\
\hat{\mathcal{K}}^{-1} \mathbb{T}(\zeta) \hat{\mathcal{K}}=\mathbb{T}\left(\mathrm{e}^{-2 \pi \mathrm{i} / r} \zeta\right), & \hat{\mathcal{K}}^{-1} \mathbb{A}_{ \pm}(\zeta) \hat{\mathcal{K}}=\mathbb{A}_{ \pm}\left(\mathrm{e}^{-2 \pi \mathrm{i} / r} \zeta\right) .
\end{array}
$$

Among others, these imply that

$$
\left[\hat{\mathcal{D}}, \mathbb{S}^{z}\right]=[\hat{\mathcal{D}}, \mathbb{H}]=[\hat{\mathcal{D}}, \mathbb{K}]=0, \quad\left[\hat{\mathcal{K}}, \mathbb{S}^{z}\right]=[\hat{\mathcal{K}}, \mathbb{H}]=[\hat{\mathcal{K}}, \mathbb{K}]=0
$$

and

$$
\begin{aligned}
& \hat{\mathcal{D}}^{-1} \mathbb{H}^{(\ell)} \hat{\mathcal{D}}=\mathbb{H}^{(\ell+1)}, \quad \hat{\mathcal{D}}^{-1} \mathbb{K}^{(\ell)} \hat{\mathcal{D}}=\mathbb{K}^{(\ell+1)}, \quad \ell=1,2, \ldots, r-1, \\
& \hat{\mathcal{D}}^{-1} \mathbb{H}^{(r)} \hat{\mathcal{D}}=\mathbb{H}^{(1)}, \quad \hat{\mathcal{D}}^{-1} \mathbb{K}^{(r)} \hat{\mathcal{D}}=\mathbb{K}^{(1)} .
\end{aligned}
$$

With $\mathbb{H}$ (6.14) taken as the Hamiltonian, the system possesses $\mathcal{Z}_{r}$ invariance (in the usual quantum mechanical sense) generated by the operator $\hat{\mathcal{D}}: \hat{\mathcal{D}}^{r}=1$. It deserves to be mentioned that in this case equation (6.8) becomes

$$
\hat{\mathcal{D}}^{-1} \boldsymbol{M}(\zeta) \hat{\mathcal{D}}=\boldsymbol{M}\left(\mathrm{e}^{+2 \pi \mathrm{i} / r} \zeta\right)
$$

With the latter at hand and taking into account that $\hat{\mathcal{D}}|0\rangle=|0\rangle$, it is straightforward to check that the action of $\hat{\mathcal{D}}$ on the Bethe state (2.18) is given by

$$
\hat{\mathcal{D}} \boldsymbol{\Psi}\left(\left\{\zeta_{j}\right\}\right)=\boldsymbol{\Psi}\left(\left\{\mathrm{e}^{+2 \pi \mathrm{i} / r} \zeta_{j}\right\}\right)
$$

Note that if $\left\{\zeta_{m}\right\}$ solves the Bethe ansatz equations (2.8) with $\eta_{J}=(-1)^{r} \mathrm{e}^{\frac{\mathrm{i} \pi}{r}(2 J-1)}$ then the set $\left\{\mathrm{e}^{+2 \pi \mathrm{i} / r} \zeta_{m}\right\}$ is also a solution of the same equations.

To summarize, when the $\left\{\eta_{J}\right\}$ are fixed to be as in (7.1) and with $|q|=|\omega|=1$, the inhomogeneous six-vertex model, together with the U(1) symmetry generated by the operator $\mathbb{S}^{z}$, possesses a set of discrete global symmetries whose generators are $\hat{\mathcal{D}}, \hat{\mathcal{K}}, \hat{\mathcal{C}} \hat{\mathcal{P}}$ and $\hat{\mathcal{T}}$. The latter obey the commutation relations

$$
\begin{array}{ll}
\hat{\mathcal{C}} \hat{\mathcal{P}} \hat{\mathcal{D}} \hat{\mathcal{P}}=\hat{\mathcal{D}}^{-1}, & \hat{\mathcal{T}} \hat{\mathcal{D}} \hat{\mathcal{T}}=\hat{\mathcal{D}} \\
\hat{\mathcal{C}} \hat{\mathcal{P}} \hat{\mathcal{K}} \hat{\mathcal{C}} \hat{\mathcal{P}}=\hat{\mathcal{K}}^{-1}, & \hat{\mathcal{T}} \hat{\mathcal{K}} \hat{\mathcal{T}}=\hat{\mathcal{K}} .
\end{array}
$$




\section{Examples}

Having discussed some general aspects of the lattice system, we now turn to the two simplest cases - the homogeneous and $\mathcal{Z}_{2}$ invariant models. The accent will be placed on the properties specific to these models.

\subsection{Homogeneous six-vertex model}

In the homogeneous case all the parameters $\left\{\eta_{J}\right\}$ are equal and can be set to one:

$$
\eta_{J}=1, \quad J=1, \ldots, N .
$$

Then it turns out that the operators $\mathbb{A}_{ \pm}(\zeta)$ with $\zeta$ real are Hermitian w.r.t. the standard Hermitian matrix conjugation:

$$
\left[\mathbb{A}_{ \pm}(\zeta)\right]^{\dagger}=\mathbb{A}_{ \pm}\left(\zeta^{*}\right)
$$

The latter follows from the general relation (5.8a) and the fact that the matrix $\hat{\mathbf{X}}$ (6.12), entering into the conjugation condition (5.6), becomes the identity

$$
\hat{\mathrm{X}}=\hat{\mathbf{1}} \text {. }
$$

Since the sesquilinear form corresponding to the $\dagger$-conjugation is positive definite, the eigenvalues of $\mathbb{A}_{ \pm}(\zeta)$ are polynomials in $\zeta$ with real coefficients. In consequence the set of zeroes of $A_{+}(\zeta)$,

$$
A_{+}(\zeta)=\prod_{m=1}^{M}\left(1-\zeta / \zeta_{m}\right),
$$

which solves the Bethe ansatz equations, coincides with the complex conjugated set:

$$
\left\{\zeta_{m}\right\}_{m=1}^{M}=\left\{\zeta_{m}^{*}\right\}_{m=1}^{M}
$$

In view of the relation (4.17), for the homogeneous model any Bethe state is invariant under the $\mathcal{C P} \mathcal{T}$ conjugation

$$
\hat{\mathcal{C}} \hat{\mathcal{P}} \hat{\mathcal{T}} \Psi=\Psi
$$

The normalization of the Bethe state $\boldsymbol{\Psi}$ (2.18) is different to that from [22]. In the latter, the authors consider

$$
\Psi^{\prime}=\sum_{1 \leq x_{1}<x_{2}<\cdots<x_{M} \leq N} \Psi^{\prime}\left(x_{1}, \ldots, x_{M}\right) \sigma_{x_{M}}^{-} \cdots \sigma_{x_{1}}^{-}|0\rangle
$$

with the wavefunction being

$$
\Psi^{\prime}\left(x_{1}, \ldots, x_{M}\right)=\sum_{\hat{P}} A_{\hat{P}} \mathrm{e}^{\mathrm{i} \sum_{m=1}^{M} p_{\hat{P} m} x_{m}} .
$$

Here $A_{\hat{P}}$ is the same as in (2.7) and

$$
\mathrm{e}^{\mathrm{i} p_{m}}=\frac{1+q \zeta_{m}}{q+\zeta_{m}}
$$

Notice that the function $\phi_{m}(x)(2.9)$ with $\eta_{J}=1$ may be written as

$$
\phi_{m}(x)=-\mathrm{i} q^{\frac{1}{2}} \omega^{-1} \frac{\left(q-q^{-1}\right) \zeta_{m}}{1+q \zeta_{m}} \mathrm{e}^{\mathrm{i} p_{m} x} .
$$


This way the relation between the Bethe state defined through (2.4)-(2.9) and $\mathbf{\Psi}^{\prime}$ reads

$$
\boldsymbol{\Psi}^{\prime}=\alpha\left(\zeta_{1}, \ldots, \zeta_{M}\right) \boldsymbol{\Psi}\left(\left\{\zeta_{j}\right\}\right),
$$

where

$$
\alpha\left(\zeta_{1}, \ldots, \zeta_{M}\right)=\left(-\mathrm{i} q^{-\frac{1}{2}} \mathrm{e}^{-\mathrm{i} \pi \mathrm{k}}\left(q-q^{-1}\right)\right)^{-M} A_{+}\left(-q^{-1}\right) .
$$

The norm of the Bethe state $\boldsymbol{\Psi}^{\prime}$ (8.1) w.r.t. the positive definite inner product is given by

$$
\left\|\Psi^{\prime}\right\|^{2}=\sum_{1 \leq x_{1}<x_{2}<\cdots<x_{M} \leq N}\left|\Psi^{\prime}\left(x_{1}, \ldots, x_{M}\right)\right|^{2} .
$$

The remarkable formula for this norm, originally conjectured by Gaudin, McCoy and Wu in [22] and proven in the work of Korepin [30] can be written as

$$
\left\|\boldsymbol{\Psi}^{\prime}\right\|^{2}=\left|\alpha\left(\zeta_{1}, \ldots, \zeta_{M}\right)\right|^{2} \mathfrak{K}[\boldsymbol{\Psi}] \prod_{m=1}^{M} \zeta_{m}
$$

where we use the notation $\mathfrak{K}[\Psi]$ from (2.20). Notice that the r.h.s. of the relation coincides with $\mathfrak{N}_{Y}[\boldsymbol{\Psi}]$ (5.13), where the operator $\mathbb{Y}$ is taken to be

$$
\mathbb{Y}=\mathbb{Y}^{\dagger}=\left(\mathrm{i}\left(q-q^{-1}\right)\right)^{2 \mathbb{S}^{z}-N} \mathbb{A}_{+}\left(-q^{+1}\right) \mathbb{A}_{+}\left(-q^{-1}\right) .
$$

At the same time $\left\|\boldsymbol{\Psi}^{\prime}\right\|^{2} \equiv\left(\boldsymbol{\Psi}^{\prime}, \boldsymbol{\Psi}^{\prime}\right)_{\star}$ for the $\star$-conjugation given by (5.9) with $\mathbb{Y}=\hat{\mathbf{1}}$. This way,

$$
\mathfrak{N}_{1}\left[\Psi^{\prime}\right]=\mathfrak{N}_{Y}[\Psi]
$$

The last formula illustrates a point previously touched on in Section 5 (see the paragraph below equation (5.14)). Namely, that different operators $\mathbb{Y}$ entering into the definition of the $\star$-conjugation may lead to the same Hermitian structure. Note that the local spin operators are Hermitian w.r.t. the $\dagger$-conjugation,

$$
\left(\sigma_{J}^{A}\right)^{\dagger}=\sigma_{J}^{A}, \quad A=x, y, z, \quad J=1, \ldots, N .
$$

For the $\star$-conjugation

$$
\left(\sigma_{J}^{A}\right)^{\star}=\mathbb{Y}^{-1} \sigma_{J}^{A} \mathbb{Y}
$$

with $\mathbb{Y}$ as in equation (8.2), when written explicitly in terms of the local spin operators, would result in a highly cumbersome expression.

Finally in the case of the homogeneous model, which corresponds to $r=1$ in (6.2), there is one quasi-shift operator that coincides with the one-site lattice translation:

$$
\mathbb{K}^{(1)}=\mathbb{K}=\hat{\mathcal{K}} .
$$

The Hamiltonian $\mathbb{H}^{(1)}(6.11)$ is just the $X X Z$ spin chain Hamiltonian

$$
\mathbb{H}^{(1)}=-\frac{\mathrm{i}}{q-q^{-1}} \sum_{m=1}^{N}\left(\sigma_{m}^{x} \sigma_{m+1}^{x}+\sigma_{m}^{y} \sigma_{m+1}^{y}+\Delta\left(\sigma_{m}^{z} \sigma_{m+1}^{z}-\hat{\mathbf{1}}\right)\right), \quad \Delta=\frac{1}{2}\left(q+q^{-1}\right)
$$

subject to the quasi-periodic boundary conditions

$$
\sigma_{N+1}^{x} \pm \mathrm{i} \sigma_{N+1}^{y}=\mathrm{e}^{ \pm 2 \pi \mathrm{ik}}\left(\sigma_{1}^{x} \pm \mathrm{i} \sigma_{1}^{y}\right), \quad \sigma_{N+1}^{z}=\sigma_{1}^{z} .
$$




\section{$8.2 \mathcal{Z}_{2}$ invariant model}

The model corresponds to the case when the number of lattice columns $N$ is even and the inhomogeneities are given by equation (7.1) with $r=2$ :

$$
\eta_{J}=\mathrm{i}(-1)^{J-1} \text {. }
$$

Bethe ansatz equations. For the $\mathcal{Z}_{2}$ invariant model, the algebraic system (2.8) reduces to

$$
\left(\frac{1+q^{+2} \zeta_{m}^{2}}{1+q^{-2} \zeta_{m}^{2}}\right)^{\frac{N}{2}}=-\omega^{2} q^{2 S^{z}} \prod_{j=1}^{M} \frac{\zeta_{j}-q^{+2} \zeta_{m}}{\zeta_{j}-q^{-2} \zeta_{m}}, \quad m=1,2, \ldots, M
$$

where $M=N / 2-S^{z}$. The eigenvalue of the transfer matrix (2.2) for the Bethe state (2.18) is given by ${ }^{6}$

$$
T(\zeta)=\omega^{+1} q^{+S^{z}}\left(1+q^{-2} \zeta^{2}\right)^{\frac{N}{2}} \prod_{m=1}^{M} \frac{\zeta_{m}-q^{+2} \zeta}{\zeta_{m}-\zeta}+\omega^{-1} q^{-S^{z}}\left(1+q^{+2} \zeta^{2}\right)^{\frac{N}{2}} \prod_{m=1}^{M} \frac{\zeta_{m}-q^{-2} \zeta}{\zeta_{m}-\zeta} .
$$

The parameters $q$ and $\omega$ are assumed to be unimodular, i.e.,

$$
q=\mathrm{e}^{\mathrm{i} \gamma}, \quad 0<\gamma<\pi ; \quad \omega=\mathrm{e}^{\mathrm{i} \pi \mathrm{k}}, \quad-\frac{1}{2}<\mathrm{k} \leq \frac{1}{2} .
$$

In $[8,9]$ the parameter $\gamma$ is swapped for $n$, such that

$$
\gamma=\frac{\pi}{n+2}
$$

Hamiltonians. Let's denote by $\mathbb{H}^{(+)}=\mathbb{H}^{(1)}$ and $\mathbb{H}^{(-)}=\mathbb{H}^{(2)}$ the Hamiltonians defined by equation (6.11) with $r=2$. It is straightforward to derive the following expressions:

$$
\begin{aligned}
\mathbb{H}^{(+)}= & \frac{1}{2} \tan (\gamma) \sum_{m=1}^{N} \sigma_{m}^{z} \sigma_{m+1}^{z}-\frac{1}{\sin (2 \gamma)} \sum_{m=1}^{N / 2}\left(\sigma_{2 m}^{x} \sigma_{2 m+2}^{x}+\sigma_{2 m}^{y} \sigma_{2 m+2}^{y}+\sigma_{2 m}^{z} \sigma_{2 m+2}^{z}\right) \\
& +\frac{\mathrm{i}}{2 \cos (\gamma)} \sum_{m=1}^{N / 2}\left(\left(\sigma_{2 m}^{x} \sigma_{2 m+1}^{x}+\sigma_{2 m}^{y} \sigma_{2 m+1}^{y}\right) \sigma_{2 m+2}^{z}-\sigma_{2 m}^{z}\left(\sigma_{2 m+1}^{x} \sigma_{2 m+2}^{x}+\sigma_{2 m+1}^{y} \sigma_{2 m+2}^{y}\right)\right) \\
& +\frac{N}{2} \cot (2 \gamma) \hat{\mathbf{1}}
\end{aligned}
$$

and

$$
\begin{aligned}
\mathbb{H}^{(-)}= & \frac{1}{2} \tan (\gamma) \sum_{m=1}^{N} \sigma_{m}^{z} \sigma_{m+1}^{z}-\frac{1}{\sin (2 \gamma)} \sum_{m=1}^{N / 2}\left(\sigma_{2 m-1}^{x} \sigma_{2 m+1}^{x}+\sigma_{2 m-1}^{y} \sigma_{2 m+1}^{y}+\sigma_{2 m-1}^{z} \sigma_{2 m+1}^{z}\right) \\
& +\frac{\mathrm{i}}{2 \cos (\gamma)} \sum_{m=1}^{N / 2}\left(\left(\sigma_{2 m-1}^{x} \sigma_{2 m}^{x}+\sigma_{2 m-1}^{y} \sigma_{2 m}^{y}\right) \sigma_{2 m+1}^{z}-\sigma_{2 m-1}^{z}\left(\sigma_{2 m}^{x} \sigma_{2 m+1}^{x}+\sigma_{2 m}^{y} \sigma_{2 m+1}^{y}\right)\right) \\
& +\frac{N}{2} \cot (2 \gamma) \hat{\mathbf{1}}
\end{aligned}
$$

\footnotetext{
${ }^{6}$ The transfer matrix $\mathbb{T}(\beta)$ and the Hamiltonian defined in equations (5) and (2) of [8], respectively, coincide with $\left(q+q^{-1} \zeta^{2}\right)^{-N / 2} \hat{\mathbf{V}} \mathbb{T}(\zeta) \hat{V}^{-1}, \hat{\mathrm{V}} \mathbb{H} \hat{V}^{-1}$, where $\mathbb{T}(\zeta)$ is given by $(2.2), \mathbb{H}$ is as in (8.3) below, the matrix $\hat{\mathrm{V}}=$ $\prod_{m=1}^{N} \exp \left(\frac{\mathrm{i} \pi}{4} \sigma_{2 m-1}^{z}\right)$, while $\zeta=\mathrm{e}^{-2 \beta}$.
} 
where

$$
\sigma_{N+\ell}^{x} \pm \mathrm{i} \sigma_{N+\ell}^{y}=\mathrm{e}^{ \pm 2 \pi \mathrm{ik}}\left(\sigma_{\ell}^{x} \pm \mathrm{i} \sigma_{\ell}^{y}\right), \quad \sigma_{N+\ell}^{z}=\sigma_{\ell}^{z}, \quad \ell=1,2
$$

The eigenvalues of these operators for the Bethe state (2.18) are expressed through the set $\left\{\zeta_{m}\right\}$ as

$$
\mathbb{H}^{( \pm)} \boldsymbol{\Psi}=\mathcal{E}^{( \pm)} \boldsymbol{\Psi}: \quad \mathcal{E}^{( \pm)}= \pm \sum_{m=1}^{N / 2-S^{z}} \frac{2\left(q-q^{-1}\right)}{\zeta_{m}-\zeta_{m}^{-1} \mp \mathrm{i}\left(q+q^{-1}\right)}
$$

The Hamiltonian $\mathbb{H}=\mathbb{H}^{(+)}+\mathbb{H}^{(-)}(6.14)$, in terms of the local spin operators, takes the form

$$
\begin{aligned}
\mathbb{H}= & \frac{1}{\sin (2 \gamma)} \sum_{m=1}^{N}\left(2 \sin ^{2}(\gamma) \sigma_{m}^{z} \sigma_{m+1}^{z}-\left(\sigma_{m}^{x} \sigma_{m+2}^{x}+\sigma_{m}^{y} \sigma_{m+2}^{y}+\sigma_{m}^{z} \sigma_{m+2}^{z}\right)\right. \\
& \left.+\mathrm{i} \sin (\gamma)\left(\sigma_{m}^{x} \sigma_{m+1}^{x}+\sigma_{m}^{y} \sigma_{m+1}^{y}\right)\left(\sigma_{m+2}^{z}-\sigma_{m-1}^{z}\right)\right)+N \cot (2 \gamma) \hat{\mathbf{1}} .
\end{aligned}
$$

Notice that $\mathbb{H}^{( \pm)}$and $\mathbb{H}$ are given by a sum of terms, where the local spin operators couple with their nearest and next-to-nearest neighbours only.

Quasi-shift operators. For the $\mathcal{Z}_{2}$ invariant model there are two quasi-shift operators $\mathbb{K}^{(+)} \equiv \mathbb{K}^{(1)}$ and $\mathbb{K}^{(-)} \equiv \mathbb{K}^{(2)}(6.10)$, whose product is equal to the two-site translation operator

$$
\mathbb{K}=\mathbb{K}^{(+)} \mathbb{K}^{(-)}
$$

In this case it is convenient to define (see also footnote 5)

$$
\mathbb{B}=\mathbb{K}^{(+)}\left(\mathbb{K}^{(-)}\right)^{-1}
$$

A computation based on equations (6.7) and (6.9) shows that

$$
\begin{aligned}
\mathbb{B}= & \omega^{-\sigma_{N}^{z}} \check{\boldsymbol{R}}_{N-1, N-2}(-1) \check{\boldsymbol{R}}_{N-3, N-4}(-1) \cdots \check{\boldsymbol{R}}_{1, N}(-1) \\
& \times \omega^{+\sigma_{N}^{z}} \check{\boldsymbol{R}}_{N, N-1}(-1) \check{\boldsymbol{R}}_{N-2, N-3}(-1) \cdots \check{\boldsymbol{R}}_{2,1}(-1) .
\end{aligned}
$$

This implies that $\mathbb{B}$ coincides with the transfer matrix of a homogeneous six-vertex model on the $45^{\circ}$-rotated square lattice with quasi-periodic boundary conditions (see Fig. 2). Its eigenvalues are given by

$$
\mathbb{B} \boldsymbol{\Psi}=B \boldsymbol{\Psi}: \quad B=\prod_{m=1}^{N / 2-S^{z}} \frac{\left(\zeta_{m}+\mathrm{i} q\right)\left(\zeta_{m}-\mathrm{i} q^{-1}\right)}{\left(\zeta_{m}-\mathrm{i} q\right)\left(\zeta_{m}+\mathrm{i} q^{-1}\right)} .
$$

Discrete symmetries. The generators of the $\mathcal{C}, \mathcal{P}$ and $\mathcal{T}$ conjugations are given by the formulae (4.5), (4.2) and (4.14), respectively, with $\eta_{J}=\mathrm{i}(-1)^{J-1}$. Their adjoint action on the Hamiltonians $\mathbb{H}^{( \pm)}$and quasi-shift operators $\mathbb{K}^{( \pm)}$reads as

$$
\begin{aligned}
\hat{\mathcal{C}} \hat{\mathcal{P}} \mathbb{H}^{( \pm)} \hat{\mathcal{C}} \hat{\mathcal{P}}=\mathbb{H}^{(\mp)}, & & \hat{\mathcal{T}} \mathbb{H}^{( \pm)} \hat{\mathcal{T}}=\mathbb{H}^{( \pm)}, \\
\hat{\mathcal{C}} \hat{\mathcal{P}} \mathbb{K}^{( \pm)} \hat{\mathcal{C}} \hat{\mathcal{P}}=\mathbb{K}^{(\mp)}, & & \hat{\mathcal{T}} \mathbb{K}^{( \pm)} \hat{\mathcal{T}}=\mathbb{K}^{( \pm)},
\end{aligned}
$$

while the similar relations for $\mathbb{H}$ and $\mathbb{K}$ were already quoted in equation (6.4). Also it is easy to see that

$$
\hat{\mathcal{C}} \hat{\mathcal{P}} \mathbb{B} \hat{\mathcal{C}} \hat{\mathcal{P}}=\hat{\mathcal{T}} \mathbb{B} \hat{\mathcal{T}}=\mathbb{B}
$$




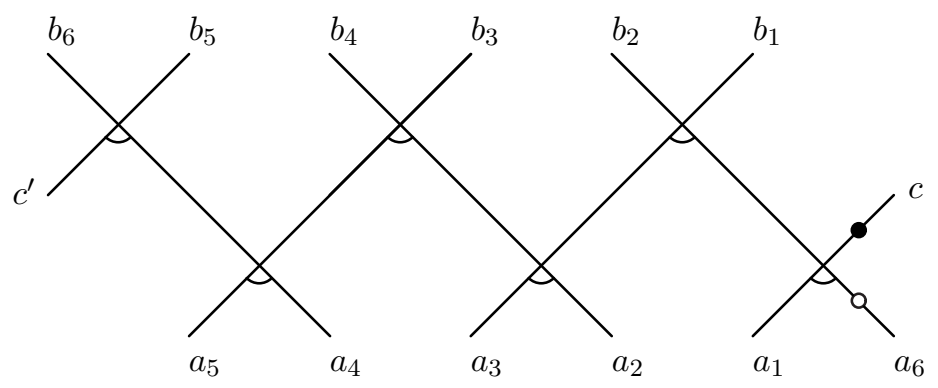

Figure 2. A graphical representation of the matrix elements $(\mathbb{B})_{a_{N} a_{N-1} \ldots a_{1}}^{b_{N-1} b_{N-1}}$ of the quasi-shift operator (8.4) for the chain of length $N=6$. Summation over the spin indices assigned to internal edges is assumed. The black and white dots correspond to $\omega^{c}$ and $\omega^{-a_{6}}$, respectively. Note that, since quasiperiodic boundary conditions are imposed, $c$ and $c^{\prime}$ should be identified.

The lattice system possesses an additional $\mathcal{Z}_{2}$ symmetry, whose generator is defined by equation (7.2) with $r=2$, i.e.,

$$
\hat{\mathcal{D}}=\prod_{m=1}^{N / 2} \check{\boldsymbol{R}}_{2 m, 2 m-1}(-1): \quad \hat{\mathcal{D}}^{2}=1 .
$$

One can show that the adjoint action of $\hat{\mathcal{D}}$ on the local spin operators is given by

$$
\begin{aligned}
& \hat{\mathcal{D}} \sigma_{m}^{ \pm} \hat{\mathcal{D}}=\frac{1}{\cos (\gamma)}\left(\sigma_{m+1}^{ \pm}-\mathrm{i} \sin (\gamma) \sigma_{m+1}^{z} \sigma_{m}^{ \pm}\right), \\
& \hat{\mathcal{D}} \sigma_{m}^{z} \hat{\mathcal{D}}=\frac{1}{\cos ^{2}(\gamma)}\left(\sigma_{m+1}^{z}-\sin ^{2}(\gamma) \sigma_{m}^{z}+2 \mathrm{i} \sin (\gamma)\left(\sigma_{m+1}^{+} \sigma_{m}^{-}+\sigma_{m+1}^{-} \sigma_{m}^{+}\right)\right)
\end{aligned}
$$

for odd values of $m$ and

$$
\begin{aligned}
& \hat{\mathcal{D}} \sigma_{m}^{ \pm} \hat{\mathcal{D}}=\frac{1}{\cos (\gamma)}\left(\sigma_{m-1}^{ \pm}+\mathrm{i} \sin (\gamma) \sigma_{m}^{ \pm} \sigma_{m-1}^{z}\right), \\
& \hat{\mathcal{D}} \sigma_{m}^{z} \hat{\mathcal{D}}=\frac{1}{\cos ^{2}(\gamma)}\left(\sigma_{m-1}^{z}-\sin ^{2}(\gamma) \sigma_{m}^{z}-2 \mathrm{i} \sin (\gamma)\left(\sigma_{m}^{+} \sigma_{m-1}^{-}+\sigma_{m}^{-} \sigma_{m-1}^{+}\right)\right)
\end{aligned}
$$

for even $m$, while

$$
\hat{\mathcal{D}}|0\rangle=|0\rangle \text {. }
$$

The commutation relations of $\hat{\mathcal{D}}$ with the operators from the commuting family are provided by equations (7.3) with $\mathrm{e}^{2 \pi \mathrm{i} / r}=-1$ and (7.4). As for the Hamiltonians $\mathbb{H}^{( \pm)}$and quasi-shift operators $\mathbb{K}^{( \pm)}$, formula (7.5) implies

$$
\hat{\mathcal{D}} \mathbb{H}^{( \pm)} \hat{\mathcal{D}}=\mathbb{H}^{(\mp)}, \quad \hat{\mathcal{D}} \mathbb{K}^{( \pm)} \hat{\mathcal{D}}=\mathbb{K}^{(\mp)}, \quad \text { whereas } \quad \hat{\mathcal{D}} \mathbb{B} \hat{\mathcal{D}}=\mathbb{B}^{-1}
$$

Hermitian structures. Consider the Hermitian matrix $\hat{X}$ entering into the conjugation condition (5.6). It is given by equation (6.12), which for $\eta_{J}=\mathrm{i}(-1)^{J-1}$ simplifies to

$$
\hat{\mathrm{X}}=\left(\prod_{J=1}^{N}\left(\eta_{J}\right)^{\frac{1}{2} \sigma_{J}^{z}}\right) \hat{\mathcal{D}}=\Sigma^{z} \hat{\mathcal{D}} \mathrm{e}^{\frac{\mathrm{i} \pi}{2}\left(\mathbb{S}^{z}-N / 2\right)}
$$

Here $\Sigma^{z}$ stands for the diagonal matrix

$$
\Sigma^{z}=\sigma_{N}^{z} \otimes \sigma_{N-2}^{z} \otimes \cdots \otimes \sigma_{2}^{z}
$$


Note that the Hermiticity of $\hat{X}$ follows from the relation

$$
\Sigma^{z} \hat{\mathcal{D}}^{\dagger} \Sigma^{z}=\mathrm{e}^{\mathrm{i} \pi\left(\mathbb{S}^{z}-N / 2\right)} \hat{\mathcal{D}}
$$

which can be easily verified using (8.5).

Unlike the homogeneous case, the matrix $\hat{X}$ is non-trivial. Among others, this implies that the operators $\mathbb{T}(\zeta)$ and $\mathbb{A}_{ \pm}(\zeta)$ are not Hermitian w.r.t. the standard matrix Hermitian conjugation. Of the Hermitian structures consistent with the integrable structure, a special rôle is played by the one defined through the $\star$-conjugation (5.9) with

$$
\mathbb{Y}=\mathrm{e}^{\mathrm{i} \pi\left(\mathbb{S}^{z}-N / 2\right)} \mathbb{A}_{+}^{(\infty)}
$$

As was already mentioned, this matrix satisfies the conditions (5.10) and (5.11). The former can also be independently checked using the relations

$$
\Sigma^{z}\left(\mathbb{A}_{+}^{(\infty)}\right)^{\dagger} \Sigma^{z}=\hat{\mathcal{D}}\left(\mathbb{A}_{+}^{(\infty)}\right)^{\dagger} \hat{\mathcal{D}}=\mathrm{e}^{\mathrm{i} \pi\left(\mathbb{S}^{z}-N / 2\right)} \mathbb{A}_{+}^{(\infty)}
$$

Recall that the "norm" of the Bethe state associated with the $\star$-conjugation for the case (8.6) is given by

$$
(\hat{\mathcal{C}} \hat{\mathcal{P}} \hat{\mathcal{T}} \Psi, \Psi)_{\star}=\mathfrak{K}[\Psi] \quad\left(\mathbb{Y}=\mathrm{e}^{\mathrm{i} \pi\left(\mathbb{S}^{z}-N / 2\right)} \mathbb{A}_{+}^{(\infty)}\right)
$$

with $\mathfrak{K}[\mathbf{\Psi}]$ being defined by equation (2.20).

The specialization of $(6.13)$ to the $\mathcal{Z}_{2}$ invariant model results in

$$
\left(\mathbb{H}^{( \pm)}\right)^{\star}=\mathbb{H}^{(\mp)}, \quad\left(\mathbb{K}^{( \pm)}\right)^{\star}=\left(\mathbb{K}^{(\mp)}\right)^{-1}
$$

and therefore

$$
\mathbb{H}^{\star}=\mathbb{H}, \quad \mathbb{K}^{\star}=\mathbb{K}^{-1}, \quad \mathbb{B}^{\star}=\mathbb{B} .
$$

\section{Conclusion}

The subject matter of this paper is the integrable inhomogeneous six-vertex model on the square lattice. The discussion is focused on various algebraic properties of the model, which are important for studying the scaling limit.

We summarized the results concerning the diagonalization problem for the transfer matrix. Explicit formulae for the Baxter $Q$-operators were presented as special transfer matrices associated with the infinite dimensional representations of the $q$-oscillator algebra.

The $\mathcal{C}, \mathcal{P}$ and $\mathcal{T}$ conjugations were introduced, and we described the constraints on the parameters of the model such that these are consistent with the integrable structure. The special features of the model possessing translational invariance were discussed. Among these, it was pointed out that the commuting family contains a set of quasi-shift operators $\mathbb{K}^{(\ell)}$ as well as Hamiltonians $\mathbb{H}^{(\ell)}$. The latter are distinguished in that they are given by a sum of "local" operators. We also formulated the conditions for which the translationally invariant model possesses an extra $\mathcal{Z}_{r}$ global symmetry and described its basic properties.

Both $\mathbb{H}^{(\ell)}$ and $\mathbb{K}^{(\ell)}$ are expected to play a key rôle for the study of the critical behaviour. The Hamiltonians are sparse matrices (most of their elements are vanishing) and there exist efficient algorithms for finding the eigenvectors and eigenvalues belonging to the low energy part of the spectrum. For a given eigenvector, it is straightforward to compute the eigenvalue of the $Q$-operators and, in turn, obtain the corresponding solution of the Bethe ansatz equations. In our subsequent paper [9] this approach is used for analyzing the scaling limit of the homogeneous 
and $\mathcal{Z}_{2}$ invariant six-vertex models. The explicit formulae collected in the last section form the starting point of that work.

For the case when both the anisotropy and twist parameters are unimodular, $|q|=|\omega|=1$, as well as when the set of inhomogeneities $\left\{\eta_{J}\right\}$ coincides with the complex conjugated set $\left\{\eta_{J}^{*}\right\}$, a family of Hermitian structures were introduced, which are consistent with the integrable one. Again, this analysis forms the preliminary set up for the study of the scaling limit of the Hermitian structures performed in [9].

Here we have only considered the square lattice models. One could also define solvable inhomogeneous six-vertex models on arbitrary planar lattices formed by intersecting straight lines [6]. Some of these are related to the quantizations of circle patterns [12] arising in the context of a discrete counterpart of the Riemann mapping theorem [15]. It would be interesting to explore the scaling limit of these constructions and their connection to CFT. Finally note that more general lattice systems with an alternating set of inhomogeneities, which still belong to the integrability class of the six-vertex model, were used in the construction of discrete integrable versions of the sine-Gordon model $[7,14,18]$.

\section{Acknowledgments}

The authors thank R.J. Baxter for providing details of the Bethe ansatz for the six-vertex model on the $45^{\circ}$-rotated square lattice [2] and N.Yu. Reshetikhin for important comments. VB acknowledges the support of the Australian Research Council grant DP180101040. The research of GK is funded by the Deutsche Forschungsgemeinschaft (DFG, German Research Foundation) under Germany's Excellence Strategy - EXC 2121 "Quantum Universe" - 390833306. The research of SL is supported by the Rutgers New High Energy Theory Center.

\section{References}

[1] Alcaraz F.C., Barber M.N., Batchelor M.T., Conformal invariance, the $X X Z$ chain and the operator content of two-dimensional critical systems, Ann. Physics 182 (1988), 280-343.

[2] Baxter R.J., Private communication, 2020.

[3] Baxter R.J., Generalized ferroelectric model on a square lattice, Stud. Appl. Math. 50 (1971), 51-69.

[4] Baxter R.J., Partition function of the eight-vertex lattice model, Ann. Physics 70 (1972), 193-228.

[5] Baxter R.J., Eight-vertex model in lattice statistics and one-dimensional anisotropic heisenberg chain. I. Some fundamental eigenvectors, Ann. Physics 76 (1973), 1-24.

[6] Baxter R.J., Solvable eight-vertex model on an arbitrary planar lattice, Philos. Trans. Roy. Soc. London Ser. A 289 (1978), 315-346.

[7] Bazhanov V., Bobenko A., Reshetikhin N., Quantum discrete sine-Gordon model at roots of 1: integrable quantum system on the integrable classical background, Comm. Math. Phys. 175 (1996), 377-400.

[8] Bazhanov V.V., Kotousov G.A., Koval S.M., Lukyanov S.L., On the scaling behaviour of the alternating spin chain, J. High Energy Phys. 2019 (2019), no. 8, 087, 30 pages, arXiv:1903.05033.

[9] Bazhanov V.V., Kotousov G.A., Koval S.M., Lukyanov S.L., Scaling limit of the $\mathcal{Z}_{2}$ invariant inhomogeneous six-vertex model, Nuclear Phys. B 965 (2021), 115337, 156 pages, arXiv:2010.10613.

[10] Bazhanov V.V., Lukyanov S.L., Zamolodchikov A.B., Integrable structure of conformal field theory. II. Q-operator and DDV equation, Comm. Math. Phys. 190 (1997), 247-278, arXiv:hep-th/9604044.

[11] Bazhanov V.V., Lukyanov S.L., Zamolodchikov A.B., Integrable structure of conformal field theory. III. The Yang-Baxter relation, Comm. Math. Phys. 200 (1999), 297-324, arXiv:hep-th/9805008.

[12] Bazhanov V.V., Mangazeev V.V., Sergeev S.M., Faddeev-Volkov solution of the Yang-Baxter equation and discrete conformal symmetry, Nuclear Phys. B $\mathbf{7 8 4}$ (2007), 234-258, arXiv:hep-th/0703041.

[13] Bethe H., Zur Theorie der Metalle. I. Eigenwerte und Eigenfunktionen der linearen Atomkette, Z. Phys. 71 (1931), 205-226.

[14] Bobenko A., Kutz N., Pinkall U., The discrete quantum pendulum, Phys. Lett. A 177 (1993), 399-404. 
[15] Bobenko A.I., Springborn B.A., Variational principles for circle patterns and Koebe's theorem, Trans. Amer. Math. Soc. 356 (2004), 659-689, arXiv:math.GT/0203250.

[16] Candu C., Ikhlef Y., Nonlinear integral equations for the $\mathrm{SL}(2, \mathbb{R}) / \mathrm{U}(1)$ black hole sigma model, J. Phys. A: Math. Theor. 46 (2013), 415401, 31 pages, arXiv:1306.2646.

[17] Destri C., de Vega H.J., Light-cone lattice approach to fermionic theories in 2D. The massive Thirring model, Nuclear Phys. B 290 (1987), 363-391.

[18] Faddeev L., Volkov A.Yu., Hirota equation as an example of an integrable symplectic map, Lett. Math. Phys. 32 (1994), 125-135, arXiv:hep-th/9405087.

[19] Faddeev L.D., Reshetikhin N.Yu., Integrability of the principal chiral field model in $1+1$ dimension, Ann. Physics 167 (1986), 227-256.

[20] Frahm H., Martins M.J., Phase diagram of an integrable alternating $\mathrm{U}_{q}[\mathfrak{s l}(2 \mid 1)]$ superspin chain, Nuclear Phys. B $\mathbf{8 6 2}$ (2012), 504-552, arXiv:1202.4676.

[21] Frahm H., Seel A., The staggered six-vertex model: conformal invariance and corrections to scaling, Nuclear Phys. B 879 (2014), 382-406, arXiv:1311.6911.

[22] Gaudin M., McCoy B.M., Wu T.T., Normalization sum for the Bethe's hypothesis wave functions of the Heisenberg-Ising chain, Phys. Rev. D 23 (1981), 417-419.

[23] Ikhlef Y., Jacobsen J., Saleur H., A staggered six-vertex model with non-compact continuum limit, Nuclear Phys. B $\mathbf{7 8 9}$ (2008), 483-524, arXiv:cond-mat/0612037.

[24] Ikhlef Y., Jacobsen J., Saleur H., The $\mathbb{Z}_{2}$ staggered vertex model and its applications, J. Phys. A: Math. Theor. 43 (2010), 225201, 37 pages, arXiv:0911.3003.

[25] Ikhlef Y., Jacobsen J., Saleur H., An integrable spin chain for the $\mathrm{SL}(2, \mathbb{R}) / \mathrm{U}(1)$ black hole sigma model, Phys. Rev. Lett. 108 (2012), 081601, 6 pages, arXiv:1109.1119.

[26] Jacobsen J.L., Saleur H., The antiferromagnetic transition for the square-lattice Potts model, Nuclear Phys. B $\mathbf{7 4 3}$ (2006), 207-248, arXiv:cond-mat/0512058.

[27] Japaridze G.I., Nersesyan A.A., Wiegmann P.B., Regularized integrable version of the one-dimensional quantum sine-Gordon model, Phys. Scripta 27 (1983), 5-7.

[28] Kadanoff L.P., Brown C.A., Correlation functions on the critical lines of the Baxter and Ashkin-Teller models, Ann. Physics 121 (1979), 318-342.

[29] Khoroshkin S.M., Tolstoy V.N., Universal R-matrix for quantized (super)algebras, Comm. Math. Phys. 141 (1991), 599-617.

[30] Korepin V.E., Calculation of norms of Bethe wave functions, Comm. Math. Phys. 86 (1982), 391-418.

[31] Lieb E.H., Residual entropy of square ice, Phys. Rev. 162 (1967), 162-172.

[32] Lieb E.H., Wu F.Y., Two-dimensional ferroelectric models, in Phase Transitions and Critical Phenomena, Editors C. Domb, M.S. Green, Academic Press, London, 1972, 331-490.

[33] Luther A., Peschel I., Calculation of critical exponents in two-dimensions from quantum field theory in one-dimension, Phys. Rev. B 12 (1975), 3908-3917.

[34] Pauling L., The structure and entropy of ice and of other crystals with some randomness of atomic arrangement, J. Amer. Chem. Soc. 57 (1935), 2680-2684.

[35] Polyakov A., Wiegmann P.B., Theory of nonabelian Goldstone bosons in two dimensions, Phys. Lett. B 131 (1983), 121-126.

[36] Reshetikhin N.Yu., Lectures on the integrability of the six-vertex model, in Exact Methods in LowDimensional Statistical Physics and Quantum Computing, Oxford University Press, Oxford, 2010, 197-266, arXiv:1010.5031.

[37] Sklyanin E.K., Takhtadzhyan L.A., Faddeev L.D., Quantum inverse problem method. I, Theoret. and Math. Phys. 40 (1979), 688-706.

[38] Sutherland B., Exact solution of a two-dimensional model for hydrogen-bonded crystals, Phys. Rev. Lett. 19 (1967), 103-104.

[39] Sutherland B., Yang C.N., Yang C.P., Exact solution of a model of two-dimensional ferroelectrics in an arbitrary external electric field, Phys. Rev. Lett. 19 (1967), 588-591.

[40] Takhtadzhan L.A., Faddeev L.D., The quantum method for the inverse problem and the Heisenberg $X Y Z$ model, Russian Math. Surveys 34 (1979), no. 5, 11-68.

[41] Yang C.P., Exact solution of a model of two-dimensional ferroelectrics in an arbitrary external electric field, Phys. Rev. Lett. 19 (1967), 586-588. 